TRANSACTIONS OF THE

AMERICAN MATHEMATICAL SOCIETY

Volume 348, Number 9, September 1996

\title{
OPTIMAL NATURAL DUALITIES. II: GENERAL THEORY
}

\author{
B. A. DAVEY AND H. A. PRIESTLEY
}

\begin{abstract}
A general theory of optimal natural dualities is presented, built on the test algebra technique introduced in an earlier paper. Given that a set $R$ of finitary algebraic relations yields a duality on a class of algebras $\mathcal{A}=\mathbb{I S P}(\underline{M})$, those subsets $R^{\prime}$ of $R$ which yield optimal dualities are characterised. Further, the manner in which the relations in $R$ are constructed from those in $R^{\prime}$ is revealed in the important special case that $\underline{M}$ generates a congruence-distributive variety and is such that each of its subalgebras is subdirectly irreducible. These results are obtained by studying a certain algebraic closure operator, called entailment, definable on any set of algebraic relations on $\underline{M}$. Applied, by way of illustration, to the variety of Kleene algebras and to the proper subvarieties $\mathbf{B}_{n}$ of pseudocomplemented distributive lattices, the theory improves upon and illuminates previous results.
\end{abstract}

\section{INTRODUCTION}

Our paper [9] claimed to open a new chapter in duality theory. This sequel, which concentrates on general theory, takes the story substantially further. We shall assume familiarity with the main strands of the plot so far; a convenient resumé can be found in [4].

Throughout we assume that we are given a quasivariety $\mathcal{A}=\mathbb{I S P}(\underline{M})$ of algebras generated by a finite algebra $\underline{M}$. We let $R \subseteq \bigcup_{n \geqslant 1} \mathbb{S}\left(\underline{M}^{n}\right)$ be a set of algebraic relations on $\underline{M}$. (An $n$-ary relation $r \subseteq M^{n}$ is called algebraic if it is the underlying set of a subalgebra of $\underline{M}^{n}$.) Define $M=(M ; R, \tau)$ to be the topological relational structure on the underlying set $M$ of $\underline{M}$ in which $\tau$ is the discrete topology. Given $\underset{\sim}{M}$, we define the category $\mathcal{X}:=\mathbb{S _ { c }} \mathbb{P}(\underset{\sim}{M})$ to have as objects all isomorphic copies of closed substructures of powers of $M$ and as morphisms the continuous $R$-preserving maps. (We shall adopt the convention that $\mathcal{A}$ includes all one-element algebras, and therefore shall include the empty structure in $\mathcal{X}$ whenever $\underline{M}$ has no one-element subalgebras.) We can then set up natural hom functors $D$ and $E$ :

$$
D=\mathcal{A}(-, \underline{M}): \mathcal{A} \rightarrow \mathcal{X} \text { and } E=\mathcal{X}(-, \underset{\sim}{M}): \mathcal{X} \rightarrow \mathcal{A} .
$$

As described in detail in [11], [4], [2], $R$ is said to yield a duality on $\mathcal{A}$ if every $A \in \mathcal{A}$ is isomorphic to its second dual $E D(A)$ (via the natural evaluation map $(\forall a \in A) e_{A}: a \mapsto e_{A}(a)$, where $\left.(\forall x \in D(A)) e_{A}(a): x \mapsto x(a)\right)$. Given an individual algebra $A \in \mathcal{A}$, we say that $R$ yields a duality on $A$ if $A \cong E D(A)$. Since we are concerned here with dualities, rather than with full or strong dualities (see [4], [1]), it suffices to take $\underset{\sim}{\sim}$ to be a purely relational structure. It is not necessary

Received by the editors August 7, 1994 and, in revised form, August 29, 1995.

1991 Mathematics Subject Classification. Primary 08B99, 06D15, 06D05, 18 A40.

Key words and phrases. Natural duality, optimal duality. 
to include full or partial operations since such operations can be replaced, for our purposes, by their graphs. We shall accordingly not distinguish between a (partial) function and its graph.

Given some finite set $R$ of finitary algebraic relations known to yield a duality on $\mathcal{A}$, it is natural to ask whether the duality is optimal in the sense that no proper subset of $R$ yields a duality. Besides being of obvious theoretical interest, this question is of practical importance in cases where general theory supplies a dualising set $R$ which is extremely large. For example, if $\underline{M}$ has a lattice reduct, it is known that $R$ can be taken to be $\mathbb{S}\left(\underline{M}^{2}\right)$; when $\underline{M}$ is the lattice $M_{3}$, then $\left|\mathbb{S}\left(\underline{M}^{2}\right)\right|=3193$. To address the question of optimality we need to be able to decide whether $R \backslash\{s\}$ fails to yield a duality on $\mathcal{A}$ for any $s \in R$, given that $R$ does yield a duality. Since our relations are required to be algebraic, every $s \in R$ has an alternative persona as a member of $\mathcal{A}$; we denote this algebra by $\underline{s}$. In [9] we made the key observation (proved in Proposition 2.2 of [9]) that in order to decide whether a given relation $s$ can be discarded from a dualising set $R$ it is sufficient to consider whether $R \backslash\{s\}$ yields a duality on the algebra $\underline{s}$, which we therefore call a test algebra. Prior to the development of this test algebra technique in [9] the search for optimal dualities was an empirical process, and in all but the simplest cases workable dualities were only discovered by taking advantage of the piggyback method of [12], [13], [6]. However, as the example of the varieties $\mathbf{B}_{n}$ of pseudocomplemented distributive lattices revealed all too clearly (see [8]), even piggyback dualities can contain unacceptably many relations. The test algebra technique was successfully applied in [9] to obtain minimal sets of relations dualising each $\mathbf{B}_{n}$. These sets are of size $O(n)$, whereas the previously known dualities contained $O(p(n))$ relations, where $p(n)$ denotes the number of partitions of the integer $n$.

Our principal aim in this paper is the characterisation of optimal dualities. The results of [9] showed that, given some finite set $\Omega$ of finitary algebraic relations known to yield a duality on $\mathcal{A}$, it was possible to use the relations in $\Omega$ in turn as test algebras to reduce $\Omega$ to a subset $R$ which yields an optimal duality. Although this process gave very satisfactory conclusions in the illustrative case of the varieties $\mathbf{B}_{n}$, as an investigation of optimality it was still incomplete. Principally dualities of piggyback type were considered and only those whose set of relations contained a generating set for the endomorphism monoid End $\underline{M}$. In the context of a general theory it is natural to start from an arbitrary finite set $\Omega$ yielding a duality and to ask not simply for a single subset $R$ yielding an optimal duality, but for a way of recognising all such subsets. Our approach to this characterisation problem relies on the analysis of entailment (previously referred to as generation; see [4], pp. 8990). The set $R$ is said to entail a relation $s$ (in symbols, $R \vdash s$ ) if for every $A \in \mathcal{A}$, every continuous map $\varphi: D(A):=\mathcal{A}(A, \underline{M}) \rightarrow M$ which preserves the relations in $R$ also preserves $s$. Entailment is intimately connected to duality. Internally, if $R$ yields a duality on $\mathcal{A}$ and $R \backslash\{s\}$ entails $s$ for some relation $s \in R$, then $R \backslash\{s\}$ yields a duality on $\mathcal{A}$, and, externally, if $R$ yields a duality on $\mathcal{A}$, then $R$ entails every algebraic relation on $\underline{M}$. Thus $R$ yields an optimal duality on $\mathcal{A}$ if and only if $R$ yields a duality on $\mathcal{A}$ and $R \backslash\{s\}$ does not entail $s$ for all $s \in R$. Locally, for a fixed $A \in \mathcal{A}$, we say $R$ entails $s$ on $X=D(A)$ if every continuous $R$-preserving map $\varphi: X \rightarrow M$ also preserves $s$. The key to the solution of the characterisation problem is the recognition that on a given set $\Omega$ of finitary algebraic relations on $\underline{M}$ the map 


$$
R \longmapsto \bar{R}:=\{s \in \Omega \mid R \vdash s\}
$$

is a closure operator (entailment closure). We can extract many riches from the lattice $\Lambda$ of all closed subsets of $\Omega$. For example, if $\Omega$ is finite and yields a duality on $\mathcal{A}$, then it is almost immediate that a subset $R$ of $\Omega$ yields an optimal duality if and only if it is a minimal set intersecting the complement of each coatom of $\Lambda$. This suggests that a careful analysis of the lattice $\Lambda$ and of its coatoms should be undertaken, and we carry this out in Section 3. Leading up to this, we investigate the entailment closure operator in detail in Section 2. Fundamental here is the recognition that the closure operator $\vdash$ is algebraic (that is, the closure of any set $R$ is the union of the closures of its finite subsets), so the lattice $\Lambda$ of closed sets is algebraic. To prove this we employ the Test Algebra Lemma, 2.3, which states that $R \vdash s$ if and only if $R \vdash s$ on $D(\underline{s})$. This result has as a corollary the aforementioned key Proposition 2.2 of [9], and should be regarded as the more fundamental assertion. An innocently simple lemma (the $r$-on- $s$ Lemma, 2.2) links the elements of $\Lambda$ with failsets, which are defined to be sets of the form

$$
\operatorname{Fail}_{\underline{s}}(u):=\{r \in \Omega \mid u \text { does not preserve } r\},
$$

where $s$ ranges over $\Omega$ and $u$ over the set of maps from $D(\underline{s})$ to $M$ which do not preserve $s$. We are able, inter alia, to describe in terms of failsets the completely meet-irreducible elements, the completely meet-prime elements and the coatoms in $\Lambda$. For example, the coatoms are the complements of those failsets which are minimal with respect to set inclusion.

In Section 4 we apply our knowledge of the lattice $\Lambda$ and of failsets in case $\Omega$ is some set yielding a duality on $\mathcal{A}$. Consider the case where $\Omega$ is finite. Since the complements of the coatoms of $\Lambda$ are precisely the minimal failsets, a subset $R$ of $\Omega$ will yield a duality on $\mathcal{A}$ if and only if it intersects every minimal failset. Thus, when $\Omega$ is reasonably small we are able, by calculating the minimal failsets, to exhibit all possible optimal dualities relative to $\Omega$.

Normally one would wish to consider dualities in which the relations are not of higher arity than necessary. In case $\underline{M}$ has a $(k+1)$-ary near-unanimity term the NU Duality Theorem ([11], Theorem 1.19, p. 142) asserts that we may take $\Omega=\bigcup_{n \leqslant k} \mathbb{S}\left(\underline{M}^{n}\right)$, and if $\underline{M}$ is a lattice-ordered algebra (so the median provides a 3-ary NU term), we may take $\Omega=\mathbb{S}(\underline{M}) \cup \mathbb{S}\left(\underline{M}^{2}\right)$, or $\Omega=\mathbb{S}\left(\underline{M}^{2}\right)$ where this proves more convenient. For the variety $\mathbf{K}$ of Kleene algebras - one of duality theory's most fruitful motivating examples - a duality was originally found, among the 13 elements of $\mathbb{S}(\underline{M}) \cup \mathbb{S}\left(\underline{M}^{2}\right)$, by experimentation ([11], pp. 176-178, or [4], p. 102). It turns out that, apart from the possible replacement of the order relation by its converse, this is essentially the unique choice if one demands relations of minimum arity. In Section 5 we sandwich between two thick slices of theory a detailed discussion of this example.

In order fully to understand optimal dualities, it is necessary, for a given relation $s$, to be able to describe the fellow members of the failsets of $s$. To make significant progress here we have to make additional assumptions. There are many examples, coming from algebraic logic, of classes, $\mathcal{A}=\mathbb{I S P}(\underline{M})$, for which useful dualities have been found. Almost all of these examples have the properties that $\mathcal{A}$ is congruencedistributive and that every subalgebra of $\underline{M}$ is subdirectly irreducible. Thus, in Section 6 , we first assume that $\underline{M}$ generates a congruence-distributive variety and 
is such that each of its subalgebras is subdirectly irreducible. This allows us to invoke the usual Pixley-Jónsson argument to assert that $s$ satisfies condition $(\mathrm{H})$, viz. that every homomorphism from $\underline{s}$ (where $\underline{s}$ is a subalgebra of $\underline{M}^{n}$ for some $n$ ) into $\underline{M}$ factorises as a projection followed by a partial endomorphism. This lures us into making our second assumption: that $\Omega$ contains the non-extendable partial endomorphisms of $\underline{M}$. While this might seem unsatisfactory, it is worth remarking that in many important examples the non-extendable partial endomorphisms may form a very small subset of the binary algebraic relations; see the comment below on the variety of de Morgan algebras. Nevertheless, the role of partial endomorphisms in duality theory remains somewhat mysterious, and deserves further investigation.

In order to describe explicitly the members of a failset $\operatorname{Fail}_{\underline{s}}(u)$ of a relation $s$ we need to be able to show how they are built from $s$ and the non-extendable partial endomorphisms. Accordingly, the problem we address is very closely related to a long-standing problem of duality theory. The Entailment Problem (alias Generation Problem) was first raised in [11], pp. 140-142, and re-stated in [4], p. 89. The Entailment Problem may be formulated as follows: find a family of constructs, each member of which involves only finitely many relations, such that if $R$ entails $s$, then $s$ can be obtained from $R$ by a finite number of applications of the constructs. In [11], pp. 140-142, a list of constructs is given which, when applied to the relations in $R$, yield relations entailed by $R$. The permitted constructs include products, intersections, domains, equalisers, kernels, and the relational product of a function $e$ and of a relation $r$. In our paper [5] we solve the Entailment Problem, and show in particular that, under the same restrictions as imposed above, namely that $R$ contains the non-extendable partial endomorphisms and that $s$ satisfies $(\mathrm{H})$, the constructs presented in [11] are sufficient.

As a finale, we return in Section 7 to the varieties $\mathbf{B}_{n}$ studied in [8], [9]. Our original account made extensive use of distributive lattice duality and is highly specific to the varieties under consideration. We are able to show how our general theory of entailment and failsets yields the principal results from [9], and thereby to understand those results at a deeper level than before.

It would be underhand to present this paper without some comments about its evolution. For both the varieties $\mathbf{S}=\mathbf{B}_{1}$ (Stone algebras) and $\mathbf{K}$ (Kleene algebras) the number of subalgebras of the square of the generating algebra is tractable-11 in each case - and it is feasible to investigate entailment by hand. For none of our other candidate test case examples would this have been possible. As has been pointed out many times before, $\mathbb{S}\left(\underline{M}^{2}\right)$ can be unpleasantly large, even when $\underline{M}$ has no more than 3 or 4 elements. For example the algebra $\underline{M}=(M ; \vee, \wedge, \sim, 0, \overline{1})$ generating the variety $\mathbf{M}$ of de Morgan algebras has $\mathbf{2}^{2}$ as its underlying lattice and $\mathbb{S}\left(\underline{M}^{2}\right)$ has 55 elements (not 45 as claimed in [11], p. 179; see [16] for a viable duality method for enumerating the subalgebras). The paper [9] would not have been written without computer output to guide us. The same is true of the present paper. The backtracking algorithm devised for [9] has since formed the basis of a library of programs for working, by duality, with finite distributive-lattice-ordered algebras (see [17]). We have used these programs extensively, to produce data, like that in Section 5, for a menagerie of varieties for which $\left|\mathbb{S}\left(\underline{M}^{2}\right)\right|$ lies between 30 and 80. We shall present in [10] an account of what we have thence been led to discover about these varieties individually and about classes (for example finitely generated quasivarieties of Heyting algebras) to which they belong. 
The authors would like to express their gratitude to the referee for detailed and helpful comments and to Miroslav Haviar and Maria Saramago for their careful reading of drafts of this paper and for constructive comments on it.

\section{Test Algebras REVISITED}

Let $\mathcal{B}$ be the set of all finitary algebraic relations on a finite algebra $\underline{M}$. We shall only consider relations which are algebraic, and these, as indicated in our remarks above concerning test algebras, we shall wish to regard both as relations and as algebras in $\mathcal{A}$. We write $s \subseteq M^{n}$ when we wish to think of $s \in \mathcal{B}$ as an $n$-ary relation, and $\underline{s} \leqslant \underline{M}^{n}$ when we regard it as an element of $\mathbb{S}\left(\underline{M}^{n}\right)$.

Each relation $s \in \mathcal{B}$ extends pointwise to powers of $M$ and then restricts to any subset of a power of $M$. Thus, in particular, $s$ has a natural pointwise interpretation as a relation on $D(A)$ for each $A \in \mathcal{A}$. Recall that a subset $R$ of $\mathcal{B}$ entails a relation $s$ on $D(A)(A \in \mathcal{A})$ if each continuous map $\varphi: D(A) \rightarrow M$ which preserves the relations in $R$ also preserves $s$, and $R$ entails $s$ (on $\mathcal{A}$ ) if it entails $s$ on $D(A)$ for each algebra $A \in \mathcal{A}$. We write $R \vdash s$ in case $R$ entails $s$.

We denote by $\pi_{i}(i=1, \ldots, n)$ the natural projection maps from $\underline{M}^{n}$ to $\underline{M}$, and, for a given subalgebra $\underline{s}$ of $\underline{M}^{n}$, let $\rho_{i}:=\left.\pi_{i}\right|_{\underline{s}}$. Note that $\rho_{1}, \ldots, \rho_{n} \in D(\underline{s})$. One further item of notation: given maps $x_{1}, \ldots, x_{n}: A \rightarrow M$ we let $x_{1} \sqcap \cdots \sqcap x_{n}: A \rightarrow$ $M^{n}$ be given by $(\forall a \in A)\left(x_{1} \sqcap \cdots \sqcap x_{n}\right)(a):=\left(x_{1}(a), \ldots, x_{n}(a)\right)$.

Lemma 2.1. Let $A \in \mathcal{A}$ and let $\underline{s} \leqslant \underline{M}^{n}$.

(a) $\left(\rho_{1}, \ldots, \rho_{n}\right) \in s_{D(\underline{s})}$, that is, $\left(\rho_{1}, \ldots, \rho_{n}\right)$ is in the relation $s$ on the set $D(\underline{s})$.

(b) Let $x_{1}, \ldots, x_{n} \in D(A)$. Then the following are equivalent (and may be interpreted as asserting that $\left(\rho_{1}, \ldots, \rho_{n}\right) \in s_{D(\underline{s})}$ is a universal instance of the relation $s$ in the class $\{D(A) \mid A \in \mathcal{A}\})$ :

(i) $\left(x_{1}, \ldots, x_{n}\right) \in s_{D(A)}$, that is, $\left(x_{1}, \ldots, x_{n}\right)$ is in the relation $s$ on the set $D(\underline{A})$

(ii) $\left(x_{1} \sqcap \cdots \sqcap x_{n}\right)(A) \subseteq s$;

(iii) there is a (necessarily unique) homomorphism $\gamma: A \rightarrow \underline{s}$, namely $\gamma=$ $x_{1} \sqcap \cdots \sqcap x_{n}$, such that $x_{i}=\rho_{i} \circ \gamma$ for all $i$;

(iv) there is a (necessarily unique) map $v: D(\underline{s}) \rightarrow D(A)$ such that $v$ preserves every finitary algebraic relation on $\underline{M}$ and $v\left(\rho_{i}\right)=x_{i}$ for all $i$;

(v) there is a (necessarily unique) map $v: D(\underline{s}) \rightarrow D(A)$ such that $v$ preserves every algebraic $n$-ary partial operation with domain $s$ and satisfies $v\left(\rho_{i}\right)=$ $x_{i}$ for all $i$;

(vi) there is an s-preserving map $v: D(\underline{s}) \rightarrow D(A)$ such that $v\left(\rho_{i}\right)=x_{i}$ for all $i$.

Proof. For (a), simply observe that $\left(\rho_{1}, \ldots, \rho_{n}\right) \in s_{D(\underline{s})}$ if and only if, for all $a \in \underline{s}$, $\left(\rho_{1} \sqcap \cdots \sqcap \rho_{n}\right)(a) \in s$. This holds because $a=\left(\rho_{1} \sqcap \cdots \sqcap \rho_{n}\right)(a)$ by definition.

We now prove (b). Assume that $\left(x_{1}, \ldots, x_{n}\right) \in s_{D(A)}$. Then, by the definition of $s_{D(A)}$, we have $\left(x_{1} \sqcap \cdots \sqcap x_{n}\right)(a)=\left(x_{1}(a), \ldots, x_{n}(a)\right) \in s$ for all $a \in A$, and hence $\left(x_{1} \sqcap \cdots \sqcap x_{n}\right)(A) \subseteq s$. Thus (i) implies (ii). If $\left(x_{1} \sqcap \cdots \sqcap x_{n}\right)(A) \subseteq s$, then the map $\gamma:=x_{1} \sqcap \cdots \sqcap x_{n}: A \rightarrow \underline{s}$ is well defined and satisfies $x_{i}=\rho_{i} \circ \gamma$ for all $i$. The categorical definition of product asserts that $\gamma$ is the unique homomorphism satisfying $x_{i}=\rho_{i} \circ \gamma$ for all $i$. Thus (ii) implies (iii).

Now assume (iii) and define $v:=D(\gamma): D(\underline{s}) \rightarrow D(A)$. Then $v$ preserves every finitary algebraic relation and $v\left(\rho_{i}\right)=D(\gamma)\left(\rho_{i}\right)=\rho_{i} \circ \gamma=x_{i}$. It remains to prove 
that $v$ is determined by its values on $\left\{\rho_{1}, \ldots, \rho_{n}\right\}$. Let $y \in D(\underline{s})$ and let $\hat{y}$ be $y$ regarded as an $n$-ary partial operation on $\underline{M}$. Thus, as $v$ preserves all finitary algebraic relations on $\underline{M}$, the map $v$ preserves $\hat{y}$. Since $\hat{y}\left(\rho_{1}, \ldots, \rho_{n}\right)=y$, we have $v(y)=v\left(\hat{y}\left(\rho_{1}, \ldots, \rho_{n}\right)\right)=\hat{y}\left(v\left(\rho_{1}\right), \ldots, v\left(\rho_{n}\right)\right)=\hat{y}\left(x_{1}, \ldots, x_{n}\right)$, whence $v$ is indeed determined by its values on $\left\{\rho_{1}, \ldots, \rho_{n}\right\}$. Thus (iii) implies (iv) and (iv) implies (v).

To prove that (v) implies (vi) note that the map $v$ guaranteed by (v) preserves the map $\rho_{1}: s \rightarrow M$ by assumption and hence preserves its domain, namely $s$. Finally we show that (vi) implies (i). Let $v$ be the map given by (vi). Since $\left(\rho_{1}, \ldots, \rho_{n}\right) \in$ $s_{D(\underline{s})}$ by (a), it follows immediately that $\left(x_{1}, \ldots, x_{n}\right)=\left(v\left(\rho_{1}\right), \ldots, v\left(\rho_{n}\right)\right) \in$ $s_{D(A)}$.

We frequently apply Lemma 2.1 when $A$ is a subalgebra of some finite power of $\underline{M}$. We accordingly state explicitly the following condensed special case of the lemma which is obtained by replacing $s$ by $r$ and $A$ by $\underline{s}$.

Corollary 2.2 (The $r$-on-s Lemma). Let $\underline{r} \leqslant \underline{M}^{m}, \underline{s} \leqslant \underline{M}^{n}$ and $x_{1}, \ldots, x_{m} \in$ $D(\underline{s})$. Then the following are equivalent:

(a) $\left(x_{1}, \ldots, x_{m}\right) \in r_{D(\underline{s})}$;

(b) there is a (necessarily unique) homomorphism, namely $\gamma=x_{1} \sqcap \cdots \sqcap x_{m}$, from $\underline{s}$ to $\underline{r}$ such that $x_{i}=\rho_{i} \circ \gamma$ for all $i$

(c) there is an r-preserving map $v: D(\underline{r}) \rightarrow D(\underline{s})$ such that $x_{i}=v\left(\rho_{i}\right)$ for all $i$.

Lemma 2.3 (The Test Algebra Lemma). Let $R \subseteq \mathcal{B}$ and let $s \in \mathcal{B}$. Then $R$ entails $s$ if and only if $R$ entails $s$ on $D(\underline{s})$.

Proof. Assume that $R$ entails $s$ on $D(\underline{s})$. Let $u: D(A) \rightarrow M$ be continuous and preserve $R$. Let $\left(x_{1}, \ldots, x_{n}\right) \in s_{D(A)}$. By Lemma 2.1 there exists an $R$-preserving map $v: D(\underline{s}) \rightarrow D(A)$ which satisfies $v\left(\rho_{i}\right)=x_{i}$ for each $i$. Hence $u \circ v: D(\underline{s}) \rightarrow M$ is $R$-preserving and so preserves $s$. Hence

$$
\left(u\left(x_{1}\right), \ldots, u\left(x_{n}\right)\right)=\left((u \circ v)\left(\rho_{1}\right), \ldots,(u \circ v)\left(\rho_{n}\right)\right) \in s,
$$

whence $u$ preserves $s$. Consequently $R \vdash s$.

Now fix a subset $\Omega$ of the set $\mathcal{B}$ of all finitary algebraic relations on $\underline{M}$. Let $s \in \Omega$ and let $u: D(\underline{s}) \rightarrow M$ be any map. Define

$$
U=\operatorname{Fail}_{\underline{s}}(u):=\{r \in \Omega \mid u \text { fails to preserve } r\} .
$$

If $u$ is an evaluation map, say $u=e_{\underline{s}}(c)$ for some $c \in \underline{s}$, then the set $U$ is empty. In case $U \neq \varnothing$ we call $U$ a weak failset of $s$ (within $\Omega$ ), and if $s \in U$ we call $U$ a failset of $s$ (within $\Omega$ ). We refer to $U$ as a failset if it is a failset of some $s \in \Omega$. It is a consequence of Corollary 3.6 below that every weak failset is a union of failsets.

It is an immediate consequence of the Test Algebra Lemma that, if $R$ is a subset of $\Omega$ and $s \in \Omega$, then $R$ entails $s$ if and only if $R$ intersects every failset of $s$. The size of the set $U$ above may be thought of as a measure of how far $u$ is from being an evaluation map. From this heuristic, it is no surprise that minimal failsets play a vital role in our theory, as each one comes from a map $u$ which is as close as possible to an evaluation map without actually being one.

We order the set of relations in a fixed failset $U$ by set inclusion, that is, $r \leqslant s$ if and only if $r$ and $s$ have the same arity and $r \subseteq s$. Since $M$ is finite, every chain in 
$U$ is finite and consequently every relation in $U$ is contained in a maximal element of $U$ and contains a minimal element of $U$.

The relationship between failsets of different relations is described in the next lemma. If $u: D(\underline{s}) \rightarrow M$ and $x_{1}, \ldots, x_{n} \in D(\underline{s})$ with $\left(x_{1}, \ldots, x_{n}\right) \in r_{D(\underline{s})}$ but $\left(u\left(x_{1}\right), \ldots, u\left(x_{n}\right)\right) \notin r$, we say that $x_{1}, \ldots, x_{n}$ witness $r \in \operatorname{Fail}_{\underline{s}}(u)$.

Lemma 2.4. Assume that $r, s \in \Omega$.

(a) If $\gamma: \underline{s} \rightarrow \underline{r}$ is a homomorphism and $u: D(\underline{s}) \rightarrow M$ is any map, then

$$
\operatorname{Fail}_{\underline{\underline{r}}}(v) \subseteq \operatorname{Fail}_{\underline{\underline{s}}}(u), \quad \text { where } v:=u \circ D(\gamma) .
$$

(b) Let $\underline{s}$ be a retract of $\underline{r}$, with associated maps $\gamma: \underline{s} \longmapsto \underline{r}$ and $\lambda: \underline{r} \rightarrow \underline{s}$ with $\lambda \circ \gamma=\mathrm{id}_{\underline{s}}$. Then, for every map $u: D(\underline{s}) \rightarrow M$,

$$
\operatorname{Fail}_{\underline{r}}(v)=\operatorname{Fail}_{\underline{s}}(u), \quad \text { where } v:=u \circ D(\gamma) .
$$

Assume further that $\Omega$ is $\mathbb{S}$-closed.

(c) Let $x_{1}, \ldots, x_{n} \in D(\underline{s})$ witness $r \in$ Fail $_{\underline{s}}(u)$; then $r^{\prime}:=\left(x_{1} \sqcap \cdots \sqcap x_{n}\right)(s) \subseteq r$ and $r^{\prime} \in \operatorname{Fail}_{\underline{s}}(u)$.

(d) Let $x_{1}, \ldots, x_{n} \in D(\underline{s})$ witness $r \in$ Fail $_{\underline{s}}(u)$ and assume that $r$ is minimal in Fail $_{\underline{s}}(u)$. Then $x_{1} \sqcap \cdots \sqcap x_{n}: \underline{s} \rightarrow \underline{r}$ is surjective.

(e) Let $x_{1}, \ldots, x_{n} \in D(\underline{s})$ witness $s \in$ Fail $_{\underline{s}}(u)$ and assume that $s$ is minimal in Fail $_{\underline{s}}(u)$. Then $x_{1} \sqcap \cdots \sqcap x_{n}: \underline{s} \rightarrow \underline{s}$ is an automorphism of $\underline{s}$.

(f) Assume that $s$ is minimal in Fail $_{\underline{s}}(u)$. Then there exists a map $v: D(\underline{s}) \rightarrow M$ such that

$$
s \in \operatorname{Fail}_{\underline{s}}(v) \subseteq \operatorname{Fail}_{\underline{s}}(u)
$$

and $s \in$ Fail $_{\underline{s}}(v)$ is witnessed by $\rho_{1}, \ldots, \rho_{n} \in D(\underline{s})$.

Proof. For any homomorphism $\gamma: \underline{s} \rightarrow \underline{r}$ the map $D(\gamma): D(\underline{r}) \rightarrow D(\underline{s})$ preserves every algebraic relation. Hence any relation preserved by $u$ is also preserved by $u \circ D(\gamma)$. Thus (a) holds.

Now consider (b). By (a), we have $\operatorname{Fail}_{\underline{r}}(v) \subseteq \operatorname{Fail}_{\underline{s}}(u)$. Let $t \in \operatorname{Fail}_{\underline{s}}(u)$ be witnessed by $x_{1}, \ldots, x_{n} \in D(\underline{s})$, and define $y_{i}:=x_{i} \circ \lambda: \underline{r} \rightarrow M$. Then

$$
\begin{aligned}
\left(y_{1} \sqcap \cdots \sqcap y_{n}\right)(r) & =\left(\left(x_{1} \circ \lambda\right) \sqcap \cdots \sqcap\left(x_{n} \circ \lambda\right)\right)(r) \\
& =\left(\left(x_{1} \sqcap \cdots \sqcap x_{n}\right) \circ \lambda\right)(r) \\
& =\left(x_{1} \sqcap \cdots \sqcap x_{n}\right)(\lambda(r)) \\
& =\left(x_{1} \sqcap \cdots \sqcap x_{n}\right)(s) \\
& \subseteq t
\end{aligned}
$$

while

$$
\begin{aligned}
\left(v\left(y_{1}\right), \ldots, v\left(y_{n}\right)\right) & =\left(u\left(y_{1} \circ \gamma\right), \ldots, u\left(y_{n} \circ \gamma\right)\right) \\
& =\left(u\left(x_{1} \circ \lambda \circ \gamma\right), \ldots, u\left(x_{n} \circ \lambda \circ \gamma\right)\right) \\
& =\left(u\left(x_{1}\right), \ldots, u\left(x_{n}\right)\right) \\
& \notin t,
\end{aligned}
$$

whence $t \in \operatorname{Fail}_{\underline{\underline{r}}}(v)$. Thus $\operatorname{Fail}_{\underline{\underline{s}}}(u) \subseteq \operatorname{Fail}_{\underline{\underline{r}}}(v)$, so (b) is proved.

Part (c) follows from the $r$-on- $s$ Lemma, 2.2, and (d) and (e) are immediate. Now assume that $s$ is minimal in $\operatorname{Fail}_{\underline{s}}(u)$ and let $s \in \operatorname{Fail}_{\underline{s}}(u)$ be witnessed by 
$x_{1}, \ldots, x_{n}$. Then, by (e), $\gamma:=x_{1} \sqcap \cdots \sqcap x_{n}$ is an automorphism of $\underline{s}$ and hence, by (a), the map $v:=u \circ D(\gamma)$ satisfies

$$
s \in \operatorname{Fail}_{\underline{s}}(v) \subseteq \operatorname{Fail}_{\underline{s}}(u) .
$$

Moreover, $\rho_{1}, \ldots, \rho_{n} \in D(\underline{s})$ witness $s \in \operatorname{Fail}_{\underline{s}}(v)$ since $\left(\rho_{1}, \ldots, \rho_{n}\right) \in s_{D(\underline{s})}$, by Lemma 2.1, while

$$
\begin{aligned}
\left(v\left(\rho_{1}\right), \ldots, v\left(\rho_{n}\right)\right) & =\left(u\left(\rho_{1} \circ \gamma\right), \ldots, u\left(\rho_{n} \circ \gamma\right)\right) \\
& =\left(u\left(x_{1}\right), \ldots, u\left(x_{n}\right)\right) \notin s .
\end{aligned}
$$

Thus (f) holds.

The test algebra technique, as originally formulated in [9], Section 2, localised to the members of a set $R$ of finitary algebraic relations, qua algebras, the question of whether $R$ yields a duality. As applied in this setting, the Test Algebra Lemma 2.3 recaptures Proposition 2.2 of [9].

Proposition 2.5. Assume that $R \subseteq \mathcal{B}$ and let $s \in \mathcal{B}$. If $R$ yields a duality on $\underline{s}$ then $R \vdash s$.

Proof. Since $R$ yields a duality on $\underline{s}$, every $R$-preserving map $u: D(\underline{s}) \rightarrow M$ is an evaluation map, and thus preserves every finitary algebraic relation, and in particular preserves $s$. Therefore $R \vdash s$ on $D(\underline{s})$, and so $R \vdash s$ by the Test Algebra Lemma.

\section{Entailment ClOSURE AND The 'TOPOLOGY' OF FAILSETS}

As before, let $\Omega \subseteq \mathcal{B}$ be a fixed set of finitary algebraic relations on $\underline{M}$. We employ the following notation concerning the failsets introduced in the previous section. For a fixed $s \in \Omega$ let

$$
\mathcal{F}_{s}:=\left\{\operatorname{Fail}_{\underline{s}}(u) \mid u: D(\underline{s}) \rightarrow M \text { fails to preserve } s\right\}
$$

be the family of all failsets of $s$ and let

$$
\mathcal{F}:=\bigcup\left\{\mathcal{F}_{s} \mid s \in \Omega\right\}
$$

be the family of all failsets.

For all $R \subseteq \Omega$ define

$$
\bar{R}:=\{s \in \Omega \mid R \vdash s\} .
$$

This is clearly a closure operator, which we shall refer to as entailment closure. The family of all closed subsets of $\Omega$ forms a complete lattice $\Lambda$. In general, entailment closure is not a topological closure operator. Notwithstanding, the family $\Lambda$ has certain 'topological' characteristics. The results of this section may be viewed as revealing connections between algebraic and 'topological' features.

The following lemma is immediate from the definitions.

Lemma 3.1. For any map $u: D(\underline{s}) \rightarrow M$, the complement of

$$
\text { Fail }_{\underline{s}}(u):=\{r \in \Omega \mid u \text { fails to preserve } r\}
$$

is closed in $\Omega$.

We can re-interpret the Test Algebra Lemma in terms of entailment closure, thereby showing that failsets serve as 'basic open sets'. 
Lemma 3.2 (The Entailment Lemma). Let $R \subseteq \Omega$ and $s \in \Omega$. Then the following are equivalent:

(a) $R \nvdash s$ (that is, $s \notin \bar{R})$;

(b) there exists $u: D(\underline{s}) \rightarrow M$ such that $s \in$ Fail $_{\underline{s}}(u) \subseteq \Omega \backslash R$.

We can refine this result to show that entailment is a finite process in that if $R$ entails $s$ then there is some finite subset $S$ of $R$ which already entails $s$. The key to the proof is the simple observation that, for any given $s \in \Omega$, the family $\mathcal{F}_{s}$ is finite. This happens because $M$ and $s$ are finite.

If $\mathcal{S}$ is a family of non-empty sets, then $T$ will be called a transversal of $\mathcal{S}$ if $T$ intersects each $U \in \mathcal{S}$ but no proper subset of $T$ does. If $\mathcal{S}$ is finite, or the sets in $\mathcal{S}$ are pairwise disjoint, then a transversal of $\mathcal{S}$ certainly exists.

Lemma 3.3. Let $R \subseteq \Omega$ and let $s \in \Omega$. The following are equivalent:

(a) $R \vdash s($ that is, $s \in \bar{R})$;

(b) $(\forall U \in \mathcal{F})(s \in U \Longrightarrow R \cap U \neq \varnothing)$;

(c) $R$ contains a transversal of $\mathcal{F}_{s}$;

(d) $S \vdash s$ for some finite subset $S$ of $R$.

Proof. It is trivial that (a) implies (b). Assume (b). Since $s \in U$ for all $U \in \mathcal{F}_{s}$ and since $\mathcal{F}_{s}$ is finite, we conclude that (c) holds. Assume that $S \subseteq R$ is a transversal of $\mathcal{F}_{s}$. Since $\mathcal{F}_{s}$ is finite, so is $S$. If $u: D(\underline{s}) \rightarrow M$ preserves $S$ then $\operatorname{Fail}_{\underline{s}}(u) \notin \mathcal{F}_{s}$ since $S$ is a transversal of $\mathcal{F}_{s}$. Hence $s \notin \operatorname{Fail}_{\underline{s}}(u)$, so $u$ preserves $s$. Thus $S \vdash s$ by the Test Algebra Lemma. The remaining implication is trivial.

The import of (a) $\Rightarrow$ (d) in Lemma 3.3 is that entailment closure is an algebraic closure operator. Consequently (see for example [7], 3.25 and 3.26) we have the following theorem.

Theorem 3.4. The lattice $\Lambda$ of closed subsets of $\Omega$ is an algebraic lattice. A closed set is compact in $\Lambda$ if and only if it is the closure of a finite set.

Any algebraic lattice is $\Lambda$-generated by its completely meet-irreducible (CMI) elements (see, for example, [7], Exercise 4.23). We next identify the completely meet-irreducible elements in $\Lambda$ in terms of failsets. Order both $\mathcal{F}_{s}$ and $\mathcal{F}$ by inclusion. If $U$ is a minimal element of $\mathcal{F}_{s}$ we call $U$ a minimal failset of $s$, and if $U$ is minimal in $\mathcal{F}$ then we refer to $U$ as a (globally) minimal failset. We shall denote the minimal failsets of $s \in \Omega$ by $\mathcal{L}_{s}$. We refer to the elements of $\mathcal{L}:=\bigcup_{s \in \Omega} \mathcal{L}_{s}$ collectively as local minimal failsets. Clearly every globally minimal failset is a local minimal failset, but not conversely. Note that, since every weak failset is a union of failsets (by 3.6 below), any weak failset $U$ which is minimal with respect to $s \in U$ is a minimal failset of $s$.

Theorem 3.5. Let $R$ be completely meet-irreducible in $\Lambda$ with $R^{*}$ its unique upper cover. Then $\Omega \backslash R$ is a minimal failset of $s$ for each $s \in R^{*} \backslash R$. Conversely if $U$ is a minimal failset of $s$ then $R:=\Omega \backslash U$ is completely meet-irreducible, with $R^{*}:=\overline{R \cup\{s\}}$ as its unique upper cover.

Proof. Assume that $R$ is completely meet-irreducible in $\Lambda$, and let $s \in R^{*} \backslash R$. Then $s \in \overline{R \cup\{r\}}$ for all $r \in \Omega \backslash R$. Since $R$ is closed and $s \notin R$, we conclude that $R$ does not entail $s$, that is,

$$
(\exists u: D(\underline{s}) \rightarrow M)\left(s \in \operatorname{Fail}_{\underline{s}}(u) \subseteq \Omega \backslash R\right) .
$$


The fact that $s \in \overline{R \cup\{r\}}$ for all $r \in \Omega \backslash R$ says that

$$
(\forall v: D(\underline{s}) \rightarrow M)\left(s \in \operatorname{Fail}_{\underline{s}}(v) \subseteq \Omega \backslash R \Longleftrightarrow \operatorname{Fail}_{\underline{s}}(v)=\Omega \backslash R\right) .
$$

Together (a) and (b) say precisely that $\Omega \backslash R=\operatorname{Fail}_{\underline{\underline{s}}}(u)$ is a minimal failset of $s$.

Conversely, assume that $U=\operatorname{Fail}_{\underline{s}}(u)$ is a minimal failset of $s$. Since by 3.1 the complement of a failset is always closed, $R:=\Omega \backslash U$ is closed and so $R \in \Lambda$. Let $r \in \Omega \backslash R$; we must show that $s \in \overline{R \cup\{r\}}$. Suppose that $s \notin \overline{R \cup\{r\}}$. Then, by the Test Algebra Lemma, there exists $v: D(\underline{s}) \rightarrow M$ which preserves $R \cup\{r\}$ but fails to preserve $s$. Thus

$$
s \in \operatorname{Fail}_{\underline{s}}(v) \subseteq U \backslash\{r\} \varsubsetneqq U=\operatorname{Fail}_{\underline{s}}(u),
$$

which contradicts the minimality of $U$ in $\mathcal{F}_{s}$.

One important consequence of this theorem is the existence of minimal failsets.

Corollary 3.6. Let $s \in \Omega$. Let $U=\operatorname{Fail}_{\underline{r}}(v)$ be a weak failset containing s. Then there is a minimal failset $U_{s}$ of $s$ with $s \in \bar{\in} U_{s} \subseteq U$.

Proof. By Lemma 3.1, $\Omega \backslash U$ is a closed set not containing $s$. Since $\Lambda$ is a topped algebraic $\bigcap$-structure, there is a closed set $R$ which is maximal with respect to not containing $s$. Clearly $R$ is completely meet-irreducible and $\overline{R \cup\{s\}}$ is its unique upper cover. Thus, by Theorem $3.5, U:=\Omega \backslash R$ is a minimal failset of $s$.

We often need to study entailment between a pair of relations.

Lemma 3.7. Let $r, s \in \Omega$. Then the following are equivalent:

(a) $r \vdash s$;

(b) $(\forall U \in \mathcal{F})(s \in U \Longrightarrow r \in U)$;

(c) $r \in \bigcap\{U \in \mathcal{F} \mid s \in U\}$;

(d) $\left(\forall U \in \mathcal{F}_{s}\right) r \in U$;

(e) $r \in \bigcap \mathcal{F}_{s}$;

(f) $r \in \bigcap \mathcal{L}_{s}$;

(g) every minimal failset of $s$ contains a minimal failset of $r$;

(h) every failset of $s$ contains a failset of $r$.

Proof. (b) $\Rightarrow$ (c) $\Rightarrow$ (d) $\Rightarrow$ (e) are trivial, while (a) $\Rightarrow$ (b) is an immediate consequence of the definition of entailment since each $U \in \mathcal{F}$ is of the form $\operatorname{Fail}_{\underline{s}}(u)$. We have (f) $\Rightarrow(\mathrm{g}) \Rightarrow(\mathrm{h})$ by 3.6 and $(\mathrm{h}) \Rightarrow$ (a) from 2.3 .

Corollary 3.8. Let $r, s \in \Omega$. Then the following are equivalent:

(a) $\overline{\{r\}}=\overline{\{s\}}$;

(b) every failset of $r$ contains a failset of $s$ and conversely;

(c) every minimal failset of $r$ is a minimal failset of $s$ and conversely.

Let $r, s \in \Omega$. If $r \vdash s$ then any failset which contains $s$ must contain $r$. Hence every failset of $s$ contains

$$
\Phi_{s}:=\{r \in \Omega \mid r \vdash s\} .
$$

The preceding lemma implies that $\Phi_{s}$ is the intersection of the (minimal) failsets of $s$. Of course, $\Phi_{s}$ is usually not itself a failset. We now investigate when it is. To do so we turn from completely meet-irreducible elements to completely meet-prime (CMP) elements. Every CMP element is CMI, but not conversely. The facts presented below in 3.9 and 3.10 are certainly well known, but are not 
well documented. In a complete lattice $L$, completely meet-prime elements and completely join-prime (CJP) elements occur in matched pairs: if $x$ is CMP, then $y:=\bigwedge\{L \backslash \downarrow x\}$ is CJP and if $y$ is CJP, $x:=\bigvee\{L \backslash \uparrow y\}$ is CMP. If $L=\downarrow x \dot{\cup} \uparrow y$ then $x$ is CMP and $y$ is CJP. Under these circumstances we shall refer to $(x, y)$ as a completely prime pair.

If $R \mapsto \bar{R}$ is a closure operator on some set $\Omega$, then we write $R \vdash s$ when $s \in \bar{R}$. We define $\Phi_{s}^{\prime}:=\Omega \backslash \Phi_{s}$. The following two lemmas refer to an arbitrary closure operator on an arbitrary set.

Lemma 3.9. Let $\Lambda$ be the lattice of closed sets of some closure operator on a set $\Omega$. A closed set $S$ is completely join-irreducible in $\Lambda$ if and only if $S=\overline{\{s\}}$ for some $s \in \Omega$ and

$$
S_{*}:=\overline{\{s\}} \cap \Phi_{s}^{\prime}=\{r \in \Omega \mid s \vdash r \text { and } r \nvdash s\}
$$

is closed.

Proof. If $S=\overline{\{s\}}$ and $S_{*}$ is closed, then $S$ is completely join-irreducible with $S_{*}$ as its unique lower cover. Assume that $S$ is completely join-irreducible. Since $S=\bigvee\{\overline{\{s\}} \mid s \in S\}$, we have $S=\overline{\{s\}}$ for some $s \in S$. Assume that $S_{*} \vdash t$. Then by the transitivity of entailment, we have $s \vdash t$. Suppose that $t \vdash s$. Then $S_{*} \vdash s$, again by transitivity of $\vdash$. Hence, since $S_{*} \subseteq \overline{\{s\}}$,

$$
\overline{\{s\}} \subseteq \bigvee\left\{\overline{\{r\}} \mid r \in S_{*}\right\}=\overline{S_{*}} \subseteq \overline{\{s\}} .
$$

As $\overline{\{s\}}$ is completely join-irreducible, we have $\overline{\{s\}}=\overline{\{r\}}$ and therefore $r \vdash s$ for some $r \in S_{*}$, which is a contradiction. Hence $t \nvdash s$ and so $t \in S_{*}$, that is, $S_{*}$ is closed.

Lemma 3.10. Let $\Lambda$ be the lattice of closed sets of some closure operator on a set $\Omega$. If $(R, S)$ is a completely prime pair, then $R=\Phi_{s}^{\prime}$ and $S=\overline{\{s\}}$ for some $s \in \Omega$. Conversely, if $\Phi_{s}^{\prime}$ is closed, then $\left(\Phi_{s}^{\prime}, \overline{\{s\}}\right)$ is a completely prime pair.

Proof. Let $(R, S)$ be a completely prime pair, and let $s \in S$, with $S=\overline{\{s\}}$. Such an $s$ exists since $S$ is CJP and therefore CJI. If $r \in R$, then $r \nvdash s$ since $R$ is closed and $s \notin R$. Thus $R \subseteq \Phi_{s}^{\prime}$. For the reverse inclusion, let $r \in \Phi_{s}^{\prime}$. Then $r \nvdash s$ and $s \notin \overline{\{r\}}$. Since $R$ is the largest closed set not containing $s$, then we conclude that $\overline{\{r\}} \subseteq R$, whence $r \in R$. Thus $\Phi_{s}^{\prime} \subseteq R$.

Conversely assume that $\Phi_{s}^{\prime}$ is closed. If $\left\{R_{i}\right\}_{i \in I}$ are closed sets such that $R_{i} \not \Phi \Phi_{s}^{\prime}$ for all $i \in I$, then for each $i \in I$ there exists $r_{i} \in R_{i}$ such that $r_{i} \vdash s$, whence $s \in R_{i}$ as $R_{i}$ is closed. Hence $s \in \bigcap_{i \in I} R_{i}$, and thus $\bigcap_{i \in I} R_{i} \nsubseteq \Phi_{s}^{\prime}$ as $s \notin \Phi_{s}^{\prime}$. Therefore $\Phi_{s}^{\prime}$ is CMP. Clearly, $\overline{\{s\}}=\bigcap\left\{T \in \Lambda \mid T \nsubseteq \Phi_{s}^{\prime}\right\}$, from which we deduce that $\left(\Phi_{s}^{\prime}, \overline{\{s\}}\right)$ is a completely prime pair.

We now apply these lemmas to the particular case of entailment closure.

Theorem 3.11. Let $s \in \Omega$. Then the following are equivalent:

(a) $\Phi_{s}$ is a failset of $s$;

(b) $\Phi_{s}$ is the unique minimal failset of $s$;

(c) $\Phi_{s}^{\prime}$ is closed;

(d) $\overline{\{s\}}$ is CJP in $\Lambda$;

(e) $\Phi_{s}^{\prime}$ is $\mathrm{CMP}$ in $\Lambda$;

(f) $\Phi_{s}^{\prime}$ is CMI in $\Lambda$;

(g) $\Phi_{s}$ is a (weak) failset. 
Proof. We have already observed that any failset which contains $s$ must contain $\Phi_{s}$. Hence (a) implies (b). Since the complement of a failset is closed, (c) follows from (b). By Lemma 3.10, (d) and (e) are equivalent and implied by (c). Finally, (e) $\Rightarrow$ (f) is trivial, while (f) $\Rightarrow$ (a) follows from Theorem 3.5. The equivalence of (a)-(f) to (g) comes from Corollary 3.6.

Note that the family $\mathcal{L}:=\bigcup_{s \in \Omega} \mathcal{L}_{s}$ of local minimal failsets is unlabelled. While we know that each member $U$ of this union is a minimal failset of the form $\operatorname{Fail}_{\underline{s}}(u)$ for some relation $s$ and some map $u$, we do not know which $s$ and which $u$. This distinction is important in practice, since it is more convenient to read and analyse output for the unlabelled family $\mathcal{L}$ than it is to keep track of the labels and so work with the corresponding labelled family. Note that Lemmas 3.3 and 3.7 allow us to check $R \vdash s$ or $r \vdash s$ from within the unlabelled family $\mathcal{L}$. From Lemma 3.7 we conclude at once that

$$
\Phi_{s}:=\{r \in \Omega \mid r \vdash s\}=\bigcap\{U \in \mathcal{L} \mid s \in U\}=\bigcap \mathcal{L}_{s} .
$$

We can give a simple algorithm to check which $U \in \mathcal{L}$ are the complements of CMPs in $\Lambda$. Form a list $\mathcal{P}$ as follows. For each $s \in \bigcup \mathcal{L}$, find $U_{s}:=\bigcap\{U \in \mathcal{L} \mid s \in U\}$. If $U_{s} \in \mathcal{L}$, then put $U_{s}$ in the list $\mathcal{P}$. Once this has been done for each $s \in \bigcup \mathcal{L}$, the list $\mathcal{P}$ consists precisely of those local minimal failsets whose complements are CMP in $\Lambda$. Alternatively, add a set $U$ to the list $\mathcal{P}$ if $U$ is the unique element of $\mathcal{L}$ which is minimal with respect to containing $s$. We can formalise this.

Theorem 3.12. Let $U$ be a subset of $\Omega$. The following are equivalent:

(a) there exists $s \in \Omega$ such that $U$ is the unique minimal failset of $s$ (that is, there exists $s \in \Omega$ such that $\left.\mathcal{L}_{s}=\{U\}\right)$;

(b) there exists $s \in \Omega$ such that $U$ is the unique element of $\mathcal{L}$ which is minimal with respect to containing $s$;

(c) $U$ is a failset and $U=\Phi_{s}$ for some $s \in \Omega$;

(d) $\Omega \backslash U$ is CMP in $\Lambda$.

Proof. The equivalence of (a), (b) and (c) follows from (*), while the equivalence of (c) and (d) comes from Lemma 3.10 and Theorem 3.11.

It is possible to recover the relation labels from the order on the family $\mathcal{L}$ of unlabelled local minimal failsets.

Lemma 3.13. For each $s \in \bigcup \mathcal{L}$, define $U_{s}:=\bigcap\{U \in \mathcal{L} \mid s \in U\}$. Then $U \in \mathcal{L}$ is a minimal failset of $s$ if and only if $U$ is minimal in $\mathcal{L}$ with respect to $U_{s} \subseteq U$.

Proof. Let $U$ be a minimal failset of $s$. Then $s \in U$ and hence $U_{s} \subseteq U$. Suppose $V \in \mathcal{L}$ is such that $U_{s} \subseteq V$. By Corollary 3.6, there exists a minimal failset of $s$, say $W$, with $s \in W \subseteq V$. Since $U_{s}=\Phi_{s}$ is a subset of every failset $V$ which contains $s$, we in fact have $s \in U_{s} \subseteq W \subseteq V$. It follows at once that $U$ is minimal in $\mathcal{L}$ with respect to $U_{s} \subseteq U$.

Conversely, assume that $U \in \mathcal{L}$ is minimal with respect to $U_{s} \subseteq U$. Then $s \in U$ and hence, by the remark above, there exists a minimal failset $W$ of $s$ with $s \in U_{s} \subseteq W \subseteq U$. The minimality of $U$ gives $U=W$, whence $U$ is a minimal failset of $s$. 
So far we have been concerned with the minimal failsets for a given relation $s$. We now investigate the set of failsets which are globally minimal. Our next proposition shows that such sets are precisely the complements of the coatoms in the algebraic lattice $\Lambda$.

Theorem 3.14. Let $\varnothing \neq U \subseteq \Omega$. Then the following are equivalent:

(a) $\Omega \backslash U$ is a coatom in $\Lambda$;

(b) $U$ is a globally minimal failset;

(c) $U$ is a minimal failset of $r$ for all $r \in U$.

Proof. Assume that $\Omega \backslash U$ is a coatom in $\Lambda$. Since coatoms are completely meetirreducible, $U$ is a minimal failset of some $r \in \Omega$, by Theorem 3.5. If $V$ is a failset with $V \subseteq U$, then $\Omega \backslash V$ is closed, by Lemma 3.1, and $\Omega \backslash U \subseteq \Omega \backslash V$. As $\Omega \backslash U$ is a coatom, $\Omega \backslash U=\Omega \backslash V$ and so $U=V$. Thus $U$ is a minimal failset. Hence (a) implies (b).

It is an immediate consequence of Corollary 3.6 that if $U=\operatorname{Fail}_{\underline{s}}(u)$ is a minimal failset then it is a minimal failset of $r$ for each $r \in U$. Thus (b) implies (c).

Finally, assume (c). To prove that $\Omega \backslash U$ is a coatom it suffices to show that if $r, s \in U$, then $(\Omega \backslash U) \cup\{r\} \vdash s$. Thus, by 3.3, we must prove that every $V \in \mathcal{F}_{s}$ intersects $(\Omega \backslash U) \cup\{r\}$. Let $V \in \mathcal{F}_{s}$. If $V \subseteq U$, then $r \in V=U$, as $U$ is a minimal failset of $r$. If $V \nsubseteq U$, then $(\Omega \backslash U) \cap V \neq \varnothing$, as required.

The following characterisation of 'density' is important in our later discussion of optimal dualities. It is immediate from Lemma 3.3.

Theorem 3.15. Let $R \subseteq \Omega$. Then the following are equivalent:

(a) $\bar{R}=\Omega$;

(b) $R$ contains a transversal of $\mathcal{F}_{s}$ for each $s \in \Omega$;

(c) $R \cap U \neq \varnothing$ for each $U \in \mathcal{F}$.

If every failset contains a minimal failset, then the following is also equivalent to (a) $-(\mathrm{c})$ :

(d) $R \cap U \neq \varnothing$ for each minimal failset $U$.

If in addition the number of minimal failsets is finite (in particular if $\Omega$ is finite), then (a)-(d) are equivalent to

(e) $R$ contains a transversal of the minimal failsets.

\section{Optimal DUAlities AND UnAVoidable SETS}

Let $\Omega$ be as in the preceding section. Given that $\Omega$ yields a duality on $\mathcal{A}$, we seek to discover which subsets $R$ of $\Omega$ yield optimal dualities, which relations in $\Omega$ are, in an appropriate sense, forced to occur in any optimal duality, and which relations never occur in an optimal duality. Our first result assembles criteria for $R \subseteq \Omega$ to yield a duality. It is an immediate consequence of Proposition 2.5.

Lemma 4.1. Assume that $\Omega \subseteq \mathcal{B}$ yields a duality on $\mathcal{A}$ and let $R \subseteq \Omega$. Then the following are equivalent:

(a) $R$ yields a duality on $\mathcal{A}$;

(b) $\bar{R}=\Omega$;

(c) $R \vdash s$ for each $s \in \Omega \backslash R$;

(d) $R \vdash s$ on $D(\underline{s})$ for each $s \in \Omega \backslash R$. 
We say that a subset $U$ of $\Omega$ is unavoidable (within $\Omega$ ) if whenever a subset $R$ of $\Omega$ yields a duality on $\mathcal{A}$, then $R \cap U \neq \varnothing$. The unavoidable subsets form an ordered set (under set inclusion) with $\Omega$ as its top. A subset $U$ of $\Omega$ will be called a minimal unavoidable set (within $\Omega$ ) if it is a minimal element of this ordered set.

Theorem 4.2. Assume that $\Omega$ yields a duality on $\mathcal{A}$. Then the following are equivalent.

(a) $U$ is a minimal unavoidable set within $\Omega$;

(b) $\Omega \backslash U$ is a coatom in $\Lambda$;

(c) $U$ is a globally minimal failset;

(d) $U$ is a minimal failset of $s$ for all $s \in U$;

(e) $\Omega \backslash U$ does not yield a duality on $\mathcal{A}$ but $(\Omega \backslash U) \cup\{s\}$ yields a duality on $\mathcal{A}$ for all $s \in U$;

(f) for all $s \in U$,

(i) $\Omega \backslash U$ does not yield a duality on $\underline{s}$, and

(ii) $(\Omega \backslash U) \cup\{s\}$ yields a duality on $U$.

Proof. The equivalence of (b), (c) and (d) was established in Theorem 3.14.

Notice that $U$ is unavoidable if and only if $\Omega \backslash U$ does not yield a duality on $\mathcal{A}$. Hence $U$ is a minimal unavoidable set if and only if $\Omega \backslash U$ is a maximal subset of $\Omega$ which does not yield a duality on $\mathcal{A}$. The equivalence of (a) and (e) follows.

It is immediate from (e) that $\Omega \backslash U$ is closed and that the only properly larger closed set is $\Omega$ itself. Hence (e) implies (b). Conversely, if $\Omega \backslash U$ is a coatom in $\Lambda$, then $\Omega \backslash U$ does not yield a duality on $\mathcal{A}$ and $\overline{(\Omega \backslash U) \cup\{s\}}=\Omega$. Since $\Omega$ yields a duality on $\mathcal{A}$ by assumption, $(\Omega \backslash U) \cup\{s\}$ yields a duality on $\mathcal{A}$. Hence (b) implies (e).

Assume that (e) holds. Let $s \in U$. If $\Omega \backslash U$ yields a duality on $\underline{s}$ then $\Omega \backslash U$ entails $s$ on $D(\underline{s})$. Since $(\Omega \backslash U) \cup\{s\}$ yields a duality on $\mathcal{A}$, by assumption, we would conclude that $\Omega \backslash U$ yields a duality on $\mathcal{A}$, which it doesn't. Thus $\Omega \backslash U$ does not yield a duality on $\underline{s}$. Since $(\Omega \backslash U) \cup\{s\}$ yields a duality on $\mathcal{A}$, it, of course, yields a duality on $U$. Thus (e) implies (f).

Finally, assume that (f) holds. Then, trivially, $\Omega \backslash U$ does not yield a duality on $\mathcal{A}$. Let $s \in U$. Since $(\Omega \backslash U) \cup\{s\}$ yields a duality on $U$, it follows that $(\Omega \backslash U) \cup\{s\}$ entails each $r \in U$ on $D(\underline{r})$ and hence $(\Omega \backslash U) \cup\{s\}$ yields a duality on $\mathcal{A}$, by Lemma 4.1.

We now look at unavoidable sets more globally. The following result is obtained by amalgamating Theorem 4.2 and Theorem 3.15 .

Theorem 4.3. Assume that $\Omega$ yields a duality on $\mathcal{A}$ and that $\Lambda$ is coatomic. If $R \subseteq \Omega$ yields a duality on $\mathcal{A}$, then $R$ intersects every minimal unavoidable set. Conversely, if $R$ intersects every minimal unavoidable set, then $R$ yields a duality on $\mathcal{A}$.

When $\Omega$ is finite more can be said. Recall that $R \subseteq \Omega$ is said to yield an optimal duality if $R$ yields a duality but no proper subset of $R$ does so. The following result tells us that if any subset will yield a duality on $\mathcal{A}$, then the very least which could work (namely a transversal of the minimal unavoidable sets) does work.

Theorem 4.4 (The Optimal Duality Theorem). Assume that $\Omega \subseteq \mathcal{B}$ is finite and yields a duality on $\mathcal{A}$. Then the following are equivalent:

(a) $R \subseteq \Omega$ yields an optimal duality on $\mathcal{A}$; 
(b) $R$ is a transversal of the globally minimal failsets in $\Omega$;

(c) $R$ is a transversal of the minimal unavoidable sets in $\Omega$.

Assume $\Omega$ is finite and yields a duality on $\mathcal{A}$. We remark that if we merely wish to prove that $R \subseteq \Omega$ yields a duality on $\mathcal{A}$ if and only if $R$ intersects every failset in $\Omega$, then the following straightforward proof is available. Let $R \subseteq \Omega$ intersect every globally minimal failset within $\Omega$. Take any $s \in \Omega$ and any $u: D(\underline{s}) \rightarrow M$ which preserves $R$. Then $\operatorname{Fail}_{\underline{s}}(u)=\varnothing$, since otherwise this failset would contain a relation from $R$, a contradiction. Hence $u$ is an evaluation map, and consequently $R$ yields a duality. Conversely, assume that $R$ yields a duality, and take $s \in \Omega$. Then any $u: D(\underline{s}) \rightarrow M$ preserving $R$ differs from all maps specifying the (necessarily nonempty) failsets. Hence $R$ intersects every failset, and thus intersects every globally minimal failset.

When $\Omega \subseteq \mathbb{S}(\underline{M}) \cup \mathbb{S}\left(\underline{M}^{2}\right)$, which is often the case in applications, there is a natural concept of absolutely unavoidable relation. We shall say that $s \in \Omega \subseteq$ $\mathbb{S}(\underline{M}) \cup \mathbb{S}\left(\underline{M}^{2}\right)$ is absolutely unavoidable (in $\Omega$ ) if $\Omega$ yields a duality on $\mathcal{A}$ and any subset $R$ of $\Omega$ which yields a duality on $\mathcal{A}$ must include $s$ if $s$ is unary, and must

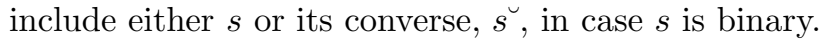

Theorem 4.5. Let $\Omega \subseteq \mathbb{S}(\underline{M}) \cup \mathbb{S}\left(\underline{M}^{2}\right)$. The following are equivalent:

(a) $s$ is absolutely unavoidable in $\Omega$;

(b) $\Omega$ yields a duality on $\mathcal{A}$ and either $\{s\}$ or $\left\{s, s^{\smile}\right\}$ is a (minimal) failset (of $s$ ) in $\Omega$.

Proof. If $s$ is unary or is a symmetric binary relation, define $V_{s}=\{s\}$, and if $s$ is a non-symmetric binary relation, define $V_{s}=\left\{s, s^{\lrcorner}\right\}$. If $s$ is absolutely unavoidable in $\Omega$, then $\Omega$ yields a duality on $\mathcal{A}$ while $\Omega \backslash V_{s}$ does not. Thus, by (e) $\Rightarrow$ (d) of Theorem $4.2, V_{s}$ is a minimal failset of $s$ and consequently must be globally minimal. Thus (a) implies (b).

Conversely, assume that $\Omega$ yields a duality on $\mathcal{A}$ and that $V_{s}$ is a minimal failset of $s$. Then, by $(\mathrm{d}) \Rightarrow(\mathrm{a})$ of Theorem $4.2, V_{s}$ is a minimal unavoidable set, whence $s$ is absolutely unavoidable.

If $\Omega$ yields a duality on $\mathcal{A}$, then $s \in \Omega$ is absolutely avoidable if whenever $R \subseteq \Omega$ is such that $R$ yields a duality on $\mathcal{A}$, then $R \backslash\{s\}$ also yields a duality on $\mathcal{A}$. That is, $s$ can always be omitted without destroying a duality. For example, all trivial relations, such as $\underline{M}, \underline{M}^{2}$ and $\Delta_{\underline{M}}$, are absolutely avoidable.

Theorem 4.6. Assume that $\Omega$ yields a duality on $\mathcal{A}$ and that $\Lambda$ is coatomic. Then the following are equivalent:

(a) $s$ is absolutely avoidable;

(b) $s$ belongs to no minimal unavoidable set;

(c) $s$ belongs to no minimal failset;

(d) $s$ belongs to the intersection of the coatoms of $\Lambda$.

Proof. The equivalence of (b), (c) and (d) is part of Theorem 4.2. Assume that $s$ is absolutely avoidable. Suppose that $s$ belongs to some minimal unavoidable set $U$. We claim that $U \backslash\{s\}$ is unavoidable, contradicting the minimality of $U$. Assume that $R$ yields a duality on $\mathcal{A}$. Then by assumption $R^{\prime}=R \backslash\{s\}$ also yields a duality on $\mathcal{A}$. Since $U$ is unavoidable, $R^{\prime}$ intersects $U$ and consequently $R$ intersects $U \backslash\{s\}$. Hence $U \backslash\{s\}$ is unavoidable, as claimed. Conversely, assume that $s$ belongs to no minimal unavoidable set and that $R \subseteq \Omega$ yields a duality on 
$\mathcal{A}$. Hence, by Theorem $3.15, R$ intersects all minimal unavoidable sets, so $R \backslash\{s\}$ does. Thus, by Theorem 4.3, $R \backslash\{s\}$ yields a duality on $\mathcal{A}$. Thus $s$ is absolutely avoidable.

\section{An interlude: the EXAmple of KleEne Algebras}

The variety $\mathbf{K}$ of Kleene algebras is $\mathbf{K}=\mathbb{I S P}(K)$, where

$$
\underline{K}=\left\langle\{0, d, 1\} ; \vee, \wedge,{ }^{\prime}, 0,1\right\rangle .
$$

The underlying lattice is the three-element chain $0<d<1$, and ' is the negation $0^{\prime}=1,1^{\prime}=0$ and $d^{\prime}=d$. This is a very important 3 -valued logic: 0 and 1 correspond to the usual Boolean truth values of "false" and "true" while $d$ corresponds to "don't know". The only proper subalgebra of $\underline{K}$ is $\underline{K}_{0}=\{0,1\}$. One may interpret $K_{0}$ as 'being in the state of knowledge' - in this state we know whether a given statement is true or false. The 'uncertainty order',

$$
\preccurlyeq=\{(0,0),(d, d),(1,1),(0, d),(1, d)\},
$$

in which 0 and 1 have minimal uncertainty and $d$ has the most uncertainty, is a subalgebra of $\underline{K}^{2}$. Since $\underline{K}$ has an underlying lattice structure, the NU Duality Theorem ([11], Theorem 1.19 , p. 142) tells us that $\Omega=\mathbb{S}\left(\underline{K}^{2}\right)$ yields a duality on $\mathbf{K}$. In line with our philosophy of using relations of minimum arity where possible we shall keep in mind also the alternative choice $\mathbb{S}(\underline{K}) \cup \mathbb{S}\left(\underline{K}^{2}\right)$ for $\Omega$.

The unary algebraic (partial) operations on $\underline{K}$ are $\Delta_{K_{0}}$ and $\Delta_{K}$, the identity maps on $\underline{K}_{0}$ and $\underline{K}$. The binary algebraic relations on $\underline{K}$ which are not graphs of (partial) maps are the products $K_{0} \times K_{0}, K_{0} \times K, K \times K_{0}, K \times K$, the order, $\preccurlyeq$, and its converse, $\succcurlyeq$, along with

$$
\begin{aligned}
& \prec:=\{(0,0),(0, d),(1, d),(1,1)\}, \\
& \succ:=\prec \smile=\{(0,0),(d, 0),(d, 1),(1,1)\}, \text { and } \\
& -:=\underline{K}^{2} \backslash\{(0,1),(1,0)\} .
\end{aligned}
$$

Figure 1(a) shows the subalgebra lattice $\left\langle\mathbb{S}\left(\underline{K}^{2}\right) ; \subseteq\right\rangle$. In it, $\prec=\preccurlyeq \cap\left(K_{0} \times K\right)$ and $\succ=\succcurlyeq \cap\left(K \times K_{0}\right)$. The meet-irreducible elements of $\mathbb{S}\left(\underline{K}^{2}\right)$ are $K \times K_{0}$, $K_{0} \times K, \preccurlyeq, \succcurlyeq$ and -. Any set which entails these will entail their intersections, and hence entail all subalgebras of $K^{2}$. As was recognised in [11], any member of the set $\left\{K_{0}, \Delta_{K_{0}}, K_{0}^{2}, K_{0} \times K, K \times K_{0}\right\}$ entails any of the others. Consequently $\left\{\preccurlyeq, K_{0},-\right\}$ entails all subalgebras of $\underline{K}^{2}$.

Let $s$ be the relation - . Recall that $\rho_{1}, \rho_{2}: s \rightarrow K$ are the restrictions of the first and second projections, respectively. It is easily seen that $D(\underline{s})$ is $\left\{\rho_{1}, \rho_{2}\right\}$, and, by Lemma 2.2(a), $\left(\rho_{1}, \rho_{2}\right) \in s_{D(\underline{s})}$ (in fact, $\left.{ }_{D(\underline{s})}=D(\underline{s})^{2}\right)$. If we define $u$ by $u\left(\rho_{1}\right)=0$ and $u\left(\rho_{2}\right)=1$, then $u$ fails to preserve - . However $u$ does preserve $\preccurlyeq$ and $\underline{K}_{0}$, since $\underline{K}_{0}$ is the empty relation and $\preccurlyeq$ is the diagonal on $D(\underline{s})$. In fact we have a much stronger statement: for $u$ as above, $\operatorname{Fail}_{\underline{s}}(u)=\{-\}$. We conclude from Theorem 4.5 that - is absolutely unavoidable. The relation - is not entailed by the members of $\underline{K}^{2} \backslash\{-\}$. Although, as subsets of $K^{2}$, the relation - coincides with the relational product $\succcurlyeq \preccurlyeq$, this identification is not valid on arbitrary spaces $D(A)$ for $A \in \mathcal{A}$. It fails in particular on $D(-)$.

The theory in Sections 3 and 4 enables us to identify all optimal dualities whose relations are of arity not more than 2 . Consider first $\Omega=\mathbb{S}\left(\underline{K}^{2}\right)$. We adopt the 


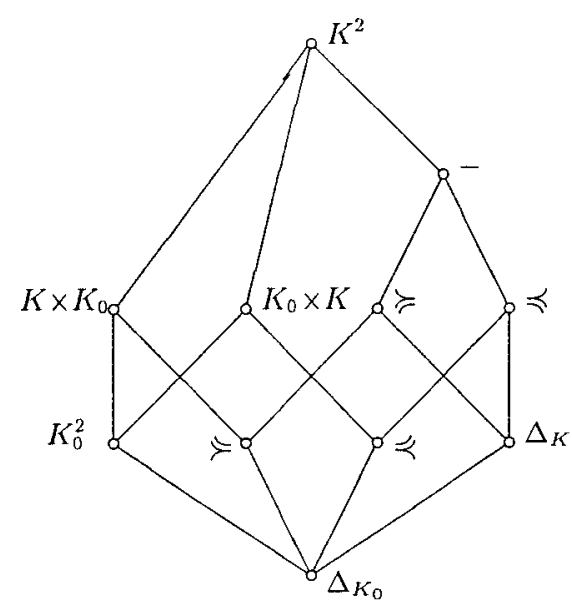

$\mathbb{S}\left(\underline{K}^{2}\right)$

(a)

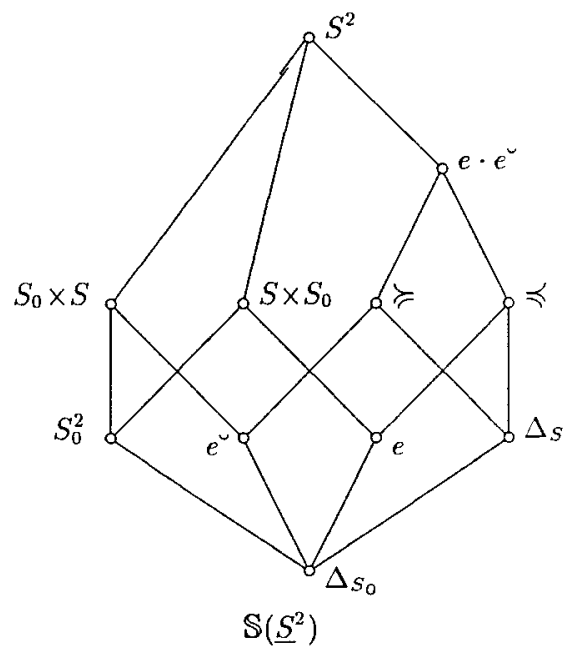

(b)

FIGURE 1

computer-generated numbering for the subalgebras of $\underline{K}^{2}$ shown in Table 1. The final column of this table lists the minimal failsets for each subalgebra of $\underline{K}^{2}$.

The globally minimal failsets are

$$
\{1,2,4,5,6,7\}, \quad\{9,10\}, \quad\{11\}
$$

note that these are, as Theorem 3.14 tells us must happen, exactly the failsets which are minimal failsets of each of their members. Their structure is further explained by the results in Section 6. In particular the failset $\{1,2,4,5,6,7\}$ arises in the manner described in Theorem 6.5. The subsets of $\mathbb{S}\left(\underline{K}^{2}\right)$ which yield optimal

\section{TABle 1}

\begin{tabular}{|l|l|l|}
\hline Subalgebra & Label & Minimal failsets \\
\hline$K_{0}^{2}$ & 1 & 124567 \\
\hline$\Delta_{K_{0}}$ & 2 & 124567 \\
\hline$\Delta_{K}$ & 3 & \\
\hline$K \times K_{0}$ & 4 & 124567 \\
\hline$K_{0} \times K$ & 5 & 124567 \\
\hline$\succ$ & 6 & $\begin{array}{l}124567 \text { and } \\
6791011\end{array}$ \\
\hline$\prec$ & 7 & $\begin{array}{l}124567 \text { and } \\
6791011\end{array}$ \\
\hline$K^{2}$ & 8 & \\
\hline$\succcurlyeq$ & 9 & 910 \\
\hline$\preccurlyeq$ & 10 & 910 \\
\hline- & 11 & 11 \\
\hline
\end{tabular}




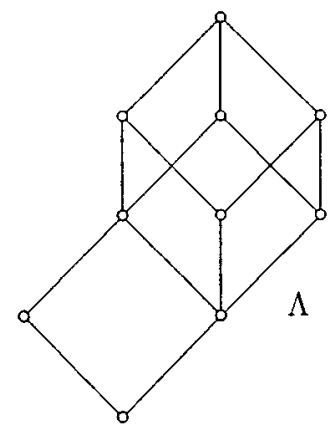

FiguRE 2

dualities are precisely the transversals of this family. The sets here are pairwise disjoint, so that we are forced to pick one element from each set. (Disjointness of the minimal failsets is not to be expected in general; see Section 7 for an example.) Relation 10 is the uncertainty order, $\preccurlyeq$, and 9 is its converse, whence $\preccurlyeq$ is absolutely unavoidable. The minimal failset $U=\{1,2,4,5,6,7\}$ consists of the binary relations which entail and are entailed by $\Delta_{K_{0}}$. At this point it is expedient to switch to $\Omega=\mathbb{S}(\underline{K}) \cup \mathbb{S}\left(\underline{K}^{2}\right)$. The effect of this change on the minimal failsets is to add $K_{0}$ to $U$; this augmented set is globally minimal. The relation $K$, in common with $\Delta_{K}$, lies in no failset. We conclude that the variety $\mathbf{K}$ has essentially only one optimal duality in which the relations are of minimum arity, namely that given by $\left\{K_{0}, \preccurlyeq,-\right\}$. In this very simple example the absolutely avoidable binary relations are just $\underline{K}^{2}$ and $\Delta_{K}$. This behaviour is not typical: generally there exist non-trivial absolutely avoidable relations (see Section 8 ).

The set $\mathcal{F}$ of minimal failsets has the following elements:

$$
\begin{array}{ll}
A=\{1,2,4,5,6,7\}, & C=\{9,10\}, \\
B=\{6,7,9,10,11\}, & D=\{11\} .
\end{array}
$$

Since the meet-irreducibles of $\Lambda$ are precisely the complements of $A, B, C$ and $D$ in $\Omega=\mathbb{S}\left(\underline{M}^{2}\right)$, the lattice $\Lambda$ is obtained (up to isomorphism) by taking the $\cup$-semilattice generated by the family of sets $\{\varnothing, A, B, C, D\}$ and ordering it by reverse inclusion. We obtain the lattice shown in Figure 2. Notice that in this case $\Lambda$ is distributive, and hence the meet-irreducible and meet-prime elements coincide. This phenomenon is rare in more complex examples.

At this point it is instructive to compare the variety of Kleene algebras with the variety of Stone algebras. For the latter we consider $\mathbb{I S P}(\underline{S})$, where $\underline{S}$ is the 3 element chain $0<d<1$ regarded as a pseudocomplemented lattice. Coincidentally, $\mathbb{S}\left(\underline{K}^{2}\right)$ and $\mathbb{S}\left(\underline{S}^{2}\right)$ are isomorphic lattices; see Figure 1. However the entailment relation works quite differently. In $\mathbb{S}\left(\underline{S}^{2}\right)$ we have (the graph of) the endomorphism $e$ mapping $d$ to 0 , and the partial order $\preccurlyeq$ has $d<1$ as its only comparability between distinct points. These two relations generate $\mathbb{S}\left(\underline{S}^{2}\right)$ as indicated in Figure 1(b). Note that $e$ entails $S_{0}$, since $S_{0}$ is the equalizer of $e$ and $\Delta_{S}$. The coatom which is not expressible as a product of subalgebras is $e \cdot e^{\iota}$. Here relational product is certainly a legitimate construct since $e$ is (the graph of) a map. Therefore all subalgebras of $\underline{S}^{2}$ are entailed by $\{\preccurlyeq, e\}$. In fact, $e \cdot e^{u}$ is absolutely avoidable. 


\section{The STRUCTURE OF FAILSETS}

The preceding section revealed examples of absolutely unavoidable relations. Such examples are relatively rare, and we were naturally led to consider minimal unavoidable sets of relations, and thence, by Theorems 4.2 and 4.4, globally minimal failsets. Since a knowledge of the globally minimal failsets, relative to a given set $\Omega$ yielding a duality on $\mathcal{A}$, tells us exactly which subsets of $\Omega$ yield optimal dualities, we look for general results which characterise certain sets as minimal failsets.

Since all $n$-ary algebraic relations in $\mathcal{B}$ can be built from the meet-irreducible elements of the lattice $\mathbb{S}\left(\underline{M}^{n}\right)$, we need to characterise such elements, and to relate them to failsets.

Let $a \in A$. A subalgebra $B$ of an algebra $A$ will be called a value of $A$ at $a$ if $B$ is maximal with respect to not containing $a$.

Lemma 6.1. $B$ is a value of $A$ if and only if $B$ is completely meet-irreducible in the lattice of subalgebras of $A$.

Proof. It is clear that if $B$ is a value at $a$ then $[B \cup\{a\}]$ is the unique upper cover of $B$ in $\mathbb{S}(A)$. (Here, and subsequently, $[S]$ denotes the subalgebra generated by a subset $S$ of $A \in \mathcal{A}$.)

Conversely, if $C$ is the unique upper cover of $B$ in $\mathbb{S}(A)$ then $B$ is a value at $a$ for each $a \in C \backslash B$.

We have already seen in Lemma 2.4(d) that the natural order on a failset has a role to play in the analysis of failsets. This order also helps us to identify subalgebras which are values.

Lemma 6.2. Let $U=$ Fail $_{\underline{s}}(u)$ be a failset.

(a) Let $r \subseteq \underline{M}^{n}$ be maximal in $U$. Then $\underline{r}$ is a value of $\underline{M}^{n}$; explicitly, if $x_{1}, \ldots, x_{n} \in D(\underline{s})$ witness $r \in \operatorname{Fail}_{\underline{s}}(u)$, then $\underline{r}$ is a value of $\underline{M}^{n}$ at $\left(u\left(x_{1}\right), \ldots\right.$, $\left.u\left(x_{n}\right)\right)$.

(b) Every element of $U$ is contained in a value in $U$. In particular, every failset contains a value.

Proof. Since $x_{1}, \ldots, x_{n} \in D(\underline{s})$ witness $r \in \operatorname{Fail}_{\underline{s}}(u)$, we have $\left(x_{1} \sqcap \cdots \sqcap x_{n}\right)(s) \subseteq$ $r$, by Lemma 2.4 , and $\left(u\left(x_{1}\right), \ldots, u\left(x_{n}\right)\right) \notin r$. Assume that $r \subseteq t \subseteq \underline{M}^{n}$ with $\left(u\left(x_{1}\right), \ldots, u\left(x_{n}\right)\right) \notin t$. Then $\left(x_{1} \sqcap \cdots \sqcap x_{n}\right)(s) \subseteq t$, and hence $x_{1}, \ldots, x_{n} \in D(\underline{s})$ witness $t \in \operatorname{Fail}_{s}(u)$. Since $r$ is maximal in $\operatorname{Fail}_{\underline{s}}(u)$, we conclude that $r=t$. Thus $r$ is a value at $\left(u\left(x_{1}\right), \ldots, u\left(x_{n}\right)\right)$. Thus (a) holds. (b) is an immediate consequence of (a).

Lemma 6.3. If $\underline{r} \leqslant \underline{M}^{n}$ is a value at $\left(a_{1}, \ldots, a_{n}\right)$ and $a_{j} \notin \pi_{j}(r)$, then $r=$ $M \times \cdots \times M \times N \times M \times \cdots \times M$, where $N=\pi_{j}(r)$, and $\underline{N}$ is a value at $a_{j}$.

Proof. Easy.

The unique minimal failsets containing $N:=\{0,1\}$ in the two examples presented in the previous section are very similar in structure. In fact, they both consist of all subalgebras of $\underline{M}^{2}$ which lie between the diagonal, $\{(a, a) \mid a \in N\}$, and $N \times M$, or between the diagonal and $M \times N$. Note that in both cases $\underline{N}$ is a value of $\underline{M}$. We are now in a position to reveal the general structure of such unique minimal failsets determined by values of $\underline{M}$. 
Let $\underline{N} \leqslant \underline{M}$ and define

$$
\Delta_{N}:=\left\{(a, a) \in M^{2} \mid a \in N\right\} .
$$

and, more generally, for $n \geqslant 1$, define

$$
\Delta_{N}^{n}:=\left\{(a, \ldots, a) \in M^{n} \mid a \in N\right\} .
$$

Now define

$$
U^{N}:=\left\{s \in \Omega \mid s \text { is } n \text {-ary and } s \cap \Delta_{M}^{n}=\Delta_{N}^{n} \text { for some } n \geqslant 1\right\} .
$$

Recall that $\Phi_{N}:=\{s \in \Omega \mid s \vdash N\}$.

Proposition 6.4. Assume that $\Omega$ contains $\mathbb{S}(\underline{M})$ and (the graphs of) the partial endomorphisms of $\underline{M}$. Let $\underline{N} \leqslant \underline{M}$ and assume that $\underline{N}$ has exactly $\ell$ upper covers in $\mathbb{S}(\underline{M})$, viz. $\left[N \cup\left\{d_{1}\right\}\right], \ldots,\left[N \cup\left\{d_{\ell}\right\}\right]$. Then the following are equivalent:

(a) the inclusion map $\alpha: \underline{N} \rightarrow \underline{M}$ is the only homomorphism from $\underline{N}$ into $\underline{M}$ (that is, $|D(\underline{N})|=1$ );

(b) $\Phi_{N}=U^{N}$ and $N$ has exactly $\ell$ minimal failsets, namely Fail ${ }_{N}\left(u_{1}\right), \ldots$, Fail $_{\underline{N}}\left(u_{\ell}\right)$, where $u_{i}: D(\underline{N}) \rightarrow M$ is the constant map onto $d_{i}$.

Proof. Assume that $D(\underline{N})=\{\alpha\}$. Firstly, note that $\operatorname{Fail}_{\underline{N}}\left(u_{i}\right) \neq \operatorname{Fail}_{\underline{N}}\left(u_{j}\right)$ for $i \neq j$, since $\left[N \cup\left\{d_{i}\right\}\right]$ is in $\operatorname{Fail}_{\underline{N}}\left(u_{j}\right)$ but not in $\operatorname{Fail}_{\underline{\underline{N}}}\left(u_{i}\right)$. Now let $\operatorname{Fail}_{\underline{\underline{N}}}(v)$ be any failset of $N$. Since $N \in \operatorname{Fail}_{\underline{N}}(\bar{v})$ and $D(\underline{N})=\{\alpha\}$, we have $d:=v(\alpha) \notin N$. Hence $[N \cup\{d\}]$ contains some cover of $\underline{N}$ in $\mathbb{S}(\underline{M})$, say $\left[N \cup\left\{d_{i}\right\}\right]$. Let $s \in \operatorname{Fail}_{\underline{N}}\left(u_{i}\right)$ and assume that $s$ is $n$-ary. Hence $(\alpha, \ldots, \alpha) \in s_{D(\underline{N})}$ and $\left(u_{i}(\alpha), \ldots, u_{i}(\alpha)\right) \notin s$, that is, $\Delta_{N}^{n} \subseteq s$ and $\bar{d}_{i}:=\left(d_{i}, \ldots, d_{i}\right) \notin s$. Suppose that $s \notin \operatorname{Fail}_{\underline{N}}(v)$. Then

$$
\begin{aligned}
(\alpha, \ldots, \alpha) \in s_{D(\underline{N})} & \Longrightarrow \bar{d}:=(v(\alpha), \ldots, v(\alpha)) \in s \\
& \Longrightarrow\left[\Delta_{N}^{n} \cup\{\bar{d}\}\right] \subseteq s \\
& \Longrightarrow\left[\Delta_{N}^{n} \cup\left\{\bar{d}_{i}\right\}\right] \subseteq s \\
& \Longrightarrow \bar{d}_{i} \in s,
\end{aligned}
$$

and this is a contradiction. It follows that $\operatorname{Fail}_{\underline{N}}\left(u_{i}\right) \subseteq \operatorname{Fail}_{\underline{N}}(v)$. Consequently, $\operatorname{Fail}_{\underline{N}}\left(u_{1}\right), \ldots, \operatorname{Fail}_{\underline{N}}\left(u_{\ell}\right)$ are the (pairwise distinct) minimal failsets of $N$. Now

$$
\begin{aligned}
s \in \bigcap_{i=1}^{\ell} \operatorname{Fail}_{\underline{N}}\left(u_{i}\right) & \Longleftrightarrow(\alpha, \ldots, \alpha) \in s \text { and }(\forall i) \bar{d}_{i} \notin s \\
& \Longleftrightarrow \Delta_{N}^{n} \subseteq s \text { and }(\forall i) \bar{d}_{i} \notin s \\
& \Longleftrightarrow \Delta_{N}^{n}=s \cap \Delta_{M}^{n} \\
& \Longleftrightarrow s \in U^{N} .
\end{aligned}
$$

(The penultimate equivalence above holds since $N=\bigcap_{i=1}^{\ell}\left[N \cup\left\{d_{i}\right\}\right]$ in $\mathbb{S}(\underline{M})$.) Since, by Lemma 3.7, $\Phi_{N}$ is the intersection of the minimal failsets of $N$, we conclude that $\Phi_{N}=U^{N}$.

Now assume (b). Let $e: \underline{N} \rightarrow \underline{M}$ be a homomorphism. If $e \in U^{N}$, then $e \cap \Delta_{M}=$ $\Delta_{N}$. Since $|e|=|N|=\left|\Delta_{N}\right|$, it follows that $e=\Delta_{N}$ as relations, whence $e=\alpha$ as maps. Hence $e \neq \alpha$ implies that $e \in \Omega \backslash U^{N}$ ( $e \in \Omega$ by hypothesis). Since $U^{N}=\Phi_{N}$, and $\Phi_{N}$ is the intersection of the minimal failsets of $N$, by (b) there exists $i$ such that $e \notin \operatorname{Fail}_{\underline{N}}\left(u_{i}\right)$. But then $u_{i}$ preserves $e$. Since $(\alpha, e) \in e_{D(\underline{N})}$ this 
forces $\left(u_{i}(\alpha), u_{i}(e)\right)=\left(d_{i}, d_{i}\right) \in e$. The latter is impossible because $d_{i} \notin \operatorname{dom} e$. We conclude that $e$ must coincide with $\alpha$.

When $\underline{N}$ is a value, we can say more.

Theorem 6.5. Assume that $\Omega$ contains $\mathbb{S}(\underline{M})$ and (the graphs of) all partial endomorphisms of $\underline{M}$. Then the following are equivalent:

(a) $U^{N}$ is a failset of $N$;

(b) $U^{N}=\Phi_{N}$ and hence is the unique minimal failset of $N$;

(c) $U^{N}$ is a failset of some relation $s \in U^{N}$;

(d) $\Omega \backslash U^{N}$ is CMP in $\Lambda$;

(e) the inclusion $\alpha$ of $\underline{N}$ into $\underline{M}$ is the only homomorphism of $\underline{N}$ into $\underline{M}$ and $\underline{N}$ is a value of $\underline{M}$.

If the assumption that $\mathbb{S}(\underline{M}) \subseteq \Omega$ is dropped, then (a) and (b) must be replaced by

(a) $U^{N}$ is a weak failset of $N$;

(b) ${ }^{\prime} U^{N}=\Phi_{\Delta_{N}}$ and hence is the unique minimal failset of $\Delta_{N}$.

Proof. First note that if $s \in U^{N}$, then $s$ entails $N$ and $\Delta_{N}$ and consequently $U^{N} \subseteq \Phi_{N}=\Phi_{\Delta_{N}}$. Hence, if $U^{N}$ is a failset of either $N$ or $\Delta_{N}$, then $U^{N}=$ $\Phi_{N}=\Phi_{\Delta_{N}}$ (since $\Phi_{s}$ is contained in every failset of $s$ ). Hence (a) is equivalent to (b). The equivalence of (b) and (d) follows from Theorem 3.11, while (b) $\Leftrightarrow$ (c) is trivial. That (a) and (b) imply and are implied by (e) follows from the preceding proposition.

If $\mathbb{S}(\underline{M}) \nsubseteq \Omega$, then define $\Omega_{1}:=\Omega \cup \mathbb{S}(\underline{M})$. Let Fail $\underline{\underline{N}}^{1}(u)$ and $U_{1}^{N}$ be the appropriate sets based on $\Omega_{1}$ rather than $\Omega$. Note that $U_{1}^{N}=\bar{U}^{N} \cup\{N\}$. Moreover, for any map $u: D(\underline{N}) \rightarrow M$ we have $U^{N}=\operatorname{Fail}_{\underline{N}}(u)$ if and only if $U_{1}^{N}=\operatorname{Fail}_{\underline{N}}^{1}(u)$, whence $U^{N}$ is a weak failset of $N$ (within $\Omega_{1}$ ) if and only if $U_{1}^{N}$ is a failset of $N$ (within $\Omega_{1}$ ). The claims regarding (a) and (b) follow easily.

Assume that $\Omega=\mathbb{S}(\underline{M}) \cup \mathbb{S}\left(\underline{M}^{2}\right)$. Referring back to the example of Kleene algebras with $\underline{M}=\underline{K}$, we note that the subalgebra $\underline{K}_{0}$ of $\underline{K}$ satisfies condition (e) and therefore all of the conditions demanded of $\underline{N}$ in Theorem 6.5. The theorem gives us exactly the failset containing $K_{0}$ presented in Section 5 . In the same way, for the variety $\mathbf{S}=\mathbb{I S P}(\underline{S})$ of Stone algebras it tells us that the unique minimal failset containing the relation $S_{0}=\{0,1\}$ consists of $S_{0}$, its products with itself and with $S$, and the non-identity endomorphism of $\underline{S}$ and its converse. In Section 7 we analyse the globally minimal failsets for the varieties $\mathbf{B}_{n}$ and show that the failset $\Phi_{\{0,1\}}$ always contains all endomorphisms which are not automorphisms.

The preceding results tell us the structure of minimal failsets of a very special type. These are important, in that globally minimal failsets of this type regularly appear in examples. Since every optimal duality is given by a transversal of the globally minimal failsets, an understanding of the structure of these sets more generally enables us to find out how different optimal dualities for the same class $\mathcal{A}$ relate to one another.

Since each failset contains a value, our objective is to identify the relations lying in the same minimal failset as a given value $\underline{s}$ of $\underline{M}^{n}$. To do this we need some means of constructing maps $u: D(\underline{s}) \rightarrow M$ which fail to preserve $s$. In order to define a map on $D(\underline{s})$ we want a workable description of the homomorphisms from $\underline{s}$ to $\underline{M}$. Of course, the maps $\rho_{i}:=\pi_{i} \uparrow_{s}(i=1, \ldots, n)$ belong to $D(\underline{s})$, as do any 
well-defined composites of these with partial endomorphisms. Let $\underline{s} \leqslant \underline{M}^{n}$ and consider the following property:

(H) every homomorphism from $\underline{s}$ to $\underline{M}$ is of the form $e \circ \rho_{i}$ for some $i \in\{1, \ldots, n\}$, where $e$ is a partial endomorphism of $\underline{M}$ and $\operatorname{im} \rho_{i} \subseteq \operatorname{dom} e$.

There is one important class of examples for which every finitary algebraic relation $s$ satisfies $(\mathrm{H})$.

Lemma 6.6. Assume that $\underline{M}$ generates a congruence-distributive variety and that every subalgebra of $\underline{M}$ is subdirectly irreducible. Then condition $(\mathrm{H})$ holds for every subalgebra of $\underline{M}^{n}$.

Proof. Let $\underline{s} \leqslant \underline{M}^{n}$ and let $x: \underline{s} \rightarrow \underline{M}$ be a homomorphism. Since im $x$ is subdirectly irreducible, the standard Pixley-Jónsson argument ([15], Theorem 2.5, [14]) says that $\operatorname{ker} \rho_{i} \leqslant \operatorname{ker} x$ for some $i$. Hence there is a partial endomorphism $e: \operatorname{im} \rho_{i} \rightarrow \underline{M}$ such that $x=e \circ \rho_{i}$, as required.

Given that $s$ satisfies condition $(\mathrm{H})$, it is natural to attempt to define a map $u: D(\underline{s}) \rightarrow M$ by choosing $\left(a_{1}, \ldots, a_{n}\right) \in \underline{M}^{n}$ and setting $u(x)=e\left(a_{i}\right)$ whenever $x=e \circ \rho_{i}$. Note that we can restrict our attention here to non-extendable partial endomorphisms $e$ of $\underline{M}$. This has the advantage of raising the probability that $u$ will be well-defined. It turns out that a dichotomy occurs. Either a well-defined map $u$ can be found, or $s$ is of one of a number of types of relation built solely from the non-extendable partial endomorphisms. Unary and binary relations are built from partial endomorphisms very simply via equalisers, joint kernels, and converses, for example. To handle relations of arbitrary arities we need to have available the constructs listed below. For a more extended discussion of the role of these constructs in duality theory, see [4] and [11].

Trivial relations. If $\theta$ is an equivalence relation on $\{1, \ldots, n\}$, then construct the trivial relation $\Delta^{\theta}:=\left\{\left(c_{1}, \ldots, c_{n}\right) \in M^{n} \mid c_{i}=c_{j} \Leftrightarrow i \theta j\right\}$.

Subscript manipulation. If $\varepsilon:\{1, \ldots, m\} \rightarrow\{1, \ldots, n\}$, then from an $m$-ary relation $r$ construct the $n$-ary relation $r^{\varepsilon}:=\left\{\left(c_{1}, \ldots, c_{n}\right) \in M^{n} \mid\left(c_{\varepsilon(1)}, \ldots, c_{\varepsilon(m)}\right) \in\right.$ $r\}$.

Trivial expansion. This is the special case of subscript manipulation in which $\varepsilon$ is one-to-one and order-preserving.

Permutation. This is the special case of subscript manipulation in which $m=n$ and $\varepsilon$ is a permutation.

Repetition removal. Assuming that $j$ is such that there exists $i$ such that $c_{i}=c_{j}$ for all $\left(c_{1}, \ldots, c_{n}\right) \in r \subseteq \underline{M}^{n}$, then from $r$ construct

$$
r_{j}^{\prime}:=\left\{\left(c_{1}, \ldots, c_{j-1}, c_{j+1}, \ldots, c_{n}\right) \in M^{n-1} \mid\left(c_{1}, \ldots, c_{n}\right) \in r\right\} .
$$

Intersection. From $n$-ary relations $r$ and $s$, construct $r \cap s$.

Product. From $r \subseteq \underline{M}^{n}$ and $s \subseteq \underline{M}^{m}$ construct

$$
r \times s:=\left\{\left(c_{1}, \ldots, c_{n}, d_{1}, \ldots, d_{m}\right) \in M^{n+m} \mid\left(c_{1}, \ldots, c_{n}\right) \in r \text { and }\left(d_{1}, \ldots, d_{m}\right) \in s\right\} .
$$

Domains. From a partial endomorphism $e: \underline{N} \rightarrow \underline{M}$, construct the domain, dom $e$, of $e$. 
Joint kernels. From partial endomorphisms $e_{1}: N_{1} \rightarrow \underline{M}$ and $e_{2}: \underline{N}_{2} \rightarrow \underline{M}$, with $\underline{N}_{1}, \underline{N}_{2} \leqslant M$, construct $\operatorname{ker}\left(e_{1}, e_{2}\right):=\left\{\left(c_{1}, c_{2}\right) \in N_{1} \times N_{2} \mid e_{1}\left(c_{1}\right)=e_{2}\left(c_{2}\right)\right\}$.

Equalisers. From partial endomorphisms $e_{1}: \underline{N}_{1} \rightarrow \underline{M}$ and $e_{2}: \underline{N}_{2} \rightarrow \underline{M}$, with $\underline{N}_{1}, \underline{N}_{2} \leqslant \underline{M}$, construct eq $\left(e_{1}, e_{2}\right):=\left\{c \in N_{1} \cap N_{2} \mid e_{1}(c)=e_{2}(c)\right\}$.

Composition. From partial endomorphisms $e_{1}: \underline{N}_{1} \rightarrow \underline{M}$ and $e_{2}: \underline{N}_{2} \rightarrow \underline{M}$, with $\underline{N}_{1}, \underline{N}_{2} \leqslant M$, construct the composite partial endomorphism $e_{1} \circ e_{2}$ with domain $\left\{c \in \operatorname{dom} e_{1} \mid e_{1}(c) \in \operatorname{dom} e_{2}\right\}$.

Action by partial endomorphisms. From $r \subseteq \underline{M}^{n}(n \geqslant 2)$ and a partial endomorphism $e: \underline{N} \rightarrow \underline{M}$, with $\underline{N} \leqslant \underline{M}$, construct

$$
e \cdot r:=\left\{\left(c_{1}, \ldots, c_{n}\right) \in M^{n} \mid c_{1} \in N \text { and }\left(e\left(c_{1}\right), c_{2}, \ldots, c_{n}\right) \in r\right\} .
$$

If $r$ is itself an endomorphism, then $e \cdot r$ is the composition of $e$ and $r$ qua maps, with $e$ done first, that is, $r \circ e$. In case $r$ is unary we define the action of $e$ on $r$ to be the natural analogue of this, namely $e^{-1}(r):=\{c \in \underline{N} \mid e(c) \in r\}$.

In the case that $\underline{M}$ is a lattice-ordered algebra, we may restrict attention to unary and binary relations. In this situation the notation simplifies considerably. Note in particular that the only non-trivial instance of subscript manipulation is

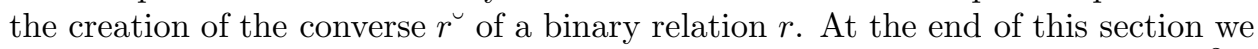
present specialisations of our general results for the case that $\Omega \subseteq \mathbb{S}(\underline{M}) \cup \mathbb{S}\left(\underline{M}^{2}\right)$.

The major results in this section stem from the following lemma.

Lemma 6.7. Let $\underline{s} \leqslant \underline{M}^{n}$ and assume that $\underline{s}$ satisfies $(\mathrm{H})$. Let $s^{*}$ be the intersection of all $n$-ary algebraic relations $r$ such that $s \subseteq r$ and $r$ has one of the following forms:

(a) $r$ is a trivial expansion of the domain of a non-extendable proper partial endomorphism,

(b) $r$ is a trivial expansion of the equaliser eq $(e, f)$ of two non-extendable partial endomorphisms $e$ and $f$ of $\underline{M}$,

(c) $r$ is a trivial expansion of the joint kernel $\operatorname{ker}(e, f)$ of two non-extendable partial endomorphisms of $\underline{M}$.

Choose $\left(a_{1}, \ldots, a_{n}\right) \in \underline{M}^{n}$ and "define" $u: D(\underline{s}) \rightarrow M$ by $u(x)=e\left(a_{i}\right)$ whenever $x=e \circ \rho_{i}$ for some non-extendable partial endomorphism e such that $\operatorname{im} \rho_{i} \subseteq \operatorname{dom} e$. Then $u$ is well defined if and only if $\left(a_{1}, \ldots, a_{n}\right) \in s^{*}$. Moreover, if $\left(a_{1}, \ldots, a_{n}\right) \in s^{*}$ and $r \in$ Fail $_{\underline{s}}(u)$, then there exist a map $\varepsilon:\{1, \ldots, m\} \rightarrow\{1, \ldots, n\}$ and nonextendable partial endomorphisms $e_{1}, \ldots, e_{m}$ of $\underline{M}$ such that

$$
\begin{aligned}
s & \subseteq\left(\left(e_{1} \times \cdots \times e_{m}\right)^{-1}(r)\right)^{\varepsilon} \\
& =\left\{\left(c_{1}, \ldots, c_{n}\right) \in\left(\operatorname{dom} e_{1} \times \cdots \times \operatorname{dom} e_{m}\right)^{\varepsilon} \mid\left(e_{1}\left(c_{\varepsilon(1)}\right), \ldots, e_{m}\left(c_{\varepsilon(m)}\right)\right) \in r\right\}
\end{aligned}
$$

and

$$
\left(a_{1}, \ldots, a_{n}\right) \notin\left(\left(e_{1} \times \cdots \times e_{m}\right)^{-1}(r)\right)^{\varepsilon} .
$$

Proof. Note that $u$ is not a well-defined map if and only if one of the following three situations occurs.

(a) For some $i$ there exists a proper non-extendable partial endomorphism $e$ such that $a_{i} \notin \operatorname{dom} e$, whence $u\left(e \circ \rho_{i}\right)$ is undefined. In this case we have $s \subseteq r:=$ $M \times \cdots \times M \times \operatorname{dom} e \times M \times \cdots \times M$, but $\left(a_{1}, \ldots, a_{n}\right) \notin r$. 
(b) For some $i$ we have non-extendable partial endomorphisms $e$ and $f$ such that $e \circ \rho_{i}=f \circ \rho_{i}$ but $e\left(a_{i}\right) \neq f\left(a_{i}\right)$. Define

$$
r:=\left\{\left(c_{1}, \ldots, c_{n}\right) \mid c_{i} \in \operatorname{dom} e \cap \operatorname{dom} f \text { and } e\left(c_{i}\right)=f\left(c_{i}\right)\right\} .
$$

Then $r$ is a trivial expansion of eq $(e, f)$ and $e \circ \rho_{i}=f \circ \rho_{i}$ implies that $s \subseteq r$ while $e\left(a_{i}\right) \neq f\left(a_{i}\right)$ implies $\left(a_{1}, \ldots, a_{n}\right) \notin r$.

(c) For some $i \neq j$ we have non-extendable partial endomorphisms $e$ and $f$ such that $e \circ \rho_{i}=f \circ \rho_{j}$ but $e\left(a_{i}\right) \neq f\left(a_{j}\right)$. Clearly,

$$
r:=\left\{\left(c_{1}, \ldots, c_{n}\right) \mid c_{i} \in \operatorname{dom} e \text { and } c_{j} \in \operatorname{dom} f \text { and } e\left(c_{i}\right)=f\left(c_{j}\right)\right\}
$$

is a trivial expansion of $\operatorname{ker}(e, f)$, and $\left(a_{1}, \ldots, a_{n}\right) \notin r$.

It follows at once that $u$ is a well-defined map if and only if $\left(a_{1}, \ldots, a_{n}\right) \in s^{*}$. Now let $\left(a_{1}, \ldots, a_{n}\right) \in s^{*}$ and assume that $r \in \operatorname{Fail}_{\underline{s}}(u)$. Thus there exist $x_{1}, \ldots, x_{m} \in$ $D(\underline{s})$ with $\left(x_{1}, \ldots, x_{m}\right) \in r_{D(\underline{s})}$ but $\left(u\left(x_{1}\right), \ldots, u\left(x_{m}\right)\right) \notin r$. By assumption $(\mathrm{H})$ we have $x_{j}=e_{j} \circ \rho_{\varepsilon(j)}$, where $e_{j}$ is a non-extendable partial endomorphism of $\underline{M}$ satisfying $\operatorname{im} \rho_{\varepsilon(j)} \subseteq$ dom $e_{j}$ and $\varepsilon:\{1, \ldots, m\} \rightarrow\{1, \ldots, n\}$. Hence, by the $r$-on- $s$ Lemma,

$$
\left(e_{1} \circ \rho_{\varepsilon(1)}\right) \sqcap \cdots \sqcap\left(e_{m} \circ \rho_{\varepsilon(m)}\right)(s) \subseteq r
$$

and

$$
\left(u\left(e_{1} \circ \rho_{\varepsilon(1)}\right), \ldots, u\left(e_{m} \circ \rho_{\varepsilon(m)}\right)\right) \notin r .
$$

Define $D:=\left(\operatorname{dom} e_{1} \times \cdots \times \operatorname{dom} e_{m}\right)^{\varepsilon}$ or, more explicitly,

$$
D:=\left\{\left(c_{1}, \ldots, c_{n}\right) \in M^{n} \mid(\forall j \in\{1, \ldots, m\}) c_{\varepsilon_{j}} \in \operatorname{dom} e_{j}\right\} .
$$

Let $\bar{\rho}_{i}:=\left.\pi_{i}\right|_{D}$ for all $i \in\{1, \ldots, n\}$. Clearly $\operatorname{im} \bar{\rho}_{\varepsilon(j)} \subseteq \operatorname{dom} e_{j}$, and hence the composite $e_{j} \circ \bar{\rho}_{\varepsilon(j)}: D \rightarrow M$ is well defined. Since im $\rho_{\varepsilon(j)} \subseteq \operatorname{dom} e_{j}$ for each $j$, the composite $e_{j} \circ \rho_{\varepsilon(j)}: s \rightarrow M$ is defined and $s \subseteq D$, whence $e_{j} \circ \bar{\rho}_{\varepsilon(j)}$ is an extension of $e_{j} \circ \rho_{\varepsilon(j)}$. Also, since $u\left(e_{j} \circ \rho_{\varepsilon(j)}\right)$ is defined, and equals $e_{j}\left(\rho_{\varepsilon(j)}\right)$, we know that $a_{\varepsilon(j)} \in \operatorname{dom} e_{j}$, and hence $\left(a_{1}, \ldots, a_{n}\right) \in D$. We thus have

$$
\left(\bar{\rho}_{\varepsilon(1)} \sqcap \cdots \sqcap \bar{\rho}_{\varepsilon(m)}\right)(s) \subseteq r \text { and }\left(e_{1}\left(a_{\varepsilon(1)}\right), \ldots, e_{m}\left(a_{\varepsilon(m)}\right)\right) \notin r,
$$

which implies that

$$
s \subseteq\left(\left(e_{1} \circ \rho_{\varepsilon(1)}\right) \sqcap \cdots \sqcap\left(e_{m} \circ \rho_{\varepsilon(m)}\right)\right)^{-1}(r)
$$

and

$$
\left(a_{1}, \ldots, a_{n}\right) \notin\left(\left(e_{1} \circ \bar{\rho}_{\varepsilon(1)}\right) \sqcap \cdots \sqcap\left(e_{m} \circ \bar{\rho}_{\varepsilon(m)}\right)\right) .
$$

Finally,

$$
\begin{aligned}
s & \subseteq\left(\left(e_{1} \circ \bar{\rho}_{\varepsilon(1)}\right) \sqcap \cdots \sqcap\left(e_{m} \circ \bar{\rho}_{\varepsilon(m)}\right)\right)^{-1}(r) \\
& =\left\{\left(b_{1}, \ldots, b_{n}\right) \in\left(\operatorname{dom} e_{1} \times \cdots \times \operatorname{dom} e_{n}\right)^{\varepsilon} \mid\left(e_{1}\left(b_{\varepsilon(1)}\right), \ldots, e_{m}\left(b_{\varepsilon(m)}\right)\right) \in r\right\} \\
& =\left(\left(e_{1} \times \cdots \times e_{m}\right)^{-1}(r)\right)^{\varepsilon},
\end{aligned}
$$

as required. 
We define $F_{s}$ to be the set of all $m$-ary algebraic relations $r$ (for $m \in \mathbb{N}$ ) such that there exist a map $\varepsilon:\{1, \ldots, m\} \rightarrow\{1, \ldots, n\}$ and non-extendable partial endomorphisms $e_{1}, \ldots, e_{m}$ of $\underline{M}$ such that

$$
\begin{aligned}
s & =\left(\left(e_{1} \times \cdots \times e_{m}\right)^{-1}(r)\right)^{\varepsilon} \\
& =\left\{\left(c_{1}, \ldots, c_{n}\right) \in\left(\operatorname{dom} e_{1} \times \cdots \times \operatorname{dom} e_{m}\right)^{\varepsilon} \mid\left(e_{1}\left(c_{\varepsilon(1)}\right), \ldots, e_{m}\left(c_{\varepsilon(m)}\right)\right) \in r\right\} .
\end{aligned}
$$

Theorem 6.8 (The Failset Theorem). Let $\Omega \subseteq \mathcal{B}$ and $s \in \Omega$. Let $\underline{s} \leqslant \underline{M}^{n}$ be a value and assume that $s$ satisfies $(\mathrm{H})$. Then either

(D) $s$ is a trivial expansion of a unary relation which is a value of $\underline{M}$ and is the domain of a proper non-extendable partial endomorphism e of $\underline{M}$, or

(E) $s$ is a trivial expansion of a unary relation which is a value of $\underline{M}$ and is the equaliser, eq $(e, f)$, of non-extendable partial endomorphisms e and $f$ of $\underline{M}$, or

$(\mathrm{K}) s$ is a trivial expansion of a binary relation which is a value of $\underline{M}^{2}$ and is the joint kernel, $\operatorname{ker}(e, f)$, of non-extendable partial endomorphisms $e$ and $f$ of $\underline{M}$, or

(F) $F_{s} \cap \Omega$ is a failset of $s$, where $F_{s}$ is as defined above.

Proof. Assume that $s$ is a value at $\left(a_{1}, \ldots, a_{n}\right)$. Define $s^{*}$ as in Lemma 6.7. If $s \subseteq s^{\prime}$, where $s^{\prime}$ is a trivial expansion of the domain of a non-extendable partial endomorphism $e$ and $\left(a_{1}, \ldots, a_{n}\right) \notin s^{\prime}$, then, since $s$ is a value at $\left(a_{1}, \ldots, a_{n}\right)$, we have $s=s^{\prime}$ and so (D) holds: the domain of $e$ is clearly a value of $M$ since $s$ is a value of $\underline{M}^{n}$. A similar argument shows that if $s \subseteq s^{\prime}$ and $\left(a_{1}, \ldots, a_{n}\right) \notin s^{\prime}$, where $s^{\prime}$ is a trivial expansion of either the equaliser or the joint kernel of two non-extendable partial endomorphisms, then (E) or (K) will hold. Consequently, if (D), (E) and (K) do not apply to $s$, then $\left(a_{1}, \ldots, a_{n}\right) \in s^{*} \backslash s$. Thus, by Lemma 6.7 , we have a well-defined map $u: D(\underline{s}) \rightarrow M$ such that if $r \in \mathrm{Fail}_{\underline{s}}^{\mathcal{B}}(u)$, then there exist a map $\varepsilon:\{1, \ldots, m\} \rightarrow\{1, \ldots, n\}$ and non-extendable partial endomorphisms $e_{1}, \ldots, e_{m}$ of $\underline{M}$ such that $s \subseteq\left(\left(e_{1} \times \cdots \times e_{m}\right)^{-1}(r)\right)^{\varepsilon}$ and $\left(a_{1}, \ldots, a_{n}\right) \notin\left(\left(e_{1} \times \cdots \times e_{m}\right)^{-1}(r)\right)^{\varepsilon}$. As $\underline{s}$ is a value of $\underline{M}^{n}$, we have $s=\left(\left(e_{1} \times \cdots \times e_{m}\right)^{-1}(r)\right)^{\varepsilon}$. Note also that $s \in \operatorname{Fail}_{\underline{s}}^{\mathcal{B}}(u)$, since $\left(\rho_{1}, \ldots, \rho_{n}\right) \in s_{D(\underline{s})}$, but $\left(u\left(\rho_{1}\right), \ldots, u\left(\rho_{n}\right)\right)=\left(a_{1}, \ldots, a_{n}\right) \notin s$. This proves that Fail $_{\underline{s}}^{\mathcal{B}}(u) \subseteq F_{s}$.

Let $r \in F_{s}$. Thus $\frac{\underline{s}}{s}=\left(\left(e_{1} \times \cdots \times e_{m}\right)^{-1}(r)\right)^{\varepsilon}$, for appropriate $e_{1}, \ldots, e_{m}$ and $\varepsilon$. It follows (see the proof of Lemma 6.7) that

$$
\left(e_{1} \circ \rho_{\varepsilon(1)}\right) \sqcap \cdots \sqcap\left(e_{m} \circ \rho_{\varepsilon(m)}\right): s \rightarrow r
$$

is well-defined. Thus, by the $r$-on- $s$ Lemma,

$$
\left(e_{1} \circ \rho_{\varepsilon(1)}, \ldots, e_{m} \circ \rho_{\varepsilon(m)}\right) \in r_{D(\underline{s})},
$$

while $\left(u\left(e_{1} \circ \rho_{\varepsilon(1)}\right), \ldots, u\left(e_{m} \circ \rho_{\varepsilon(m)}\right)\right) \notin r$, since

$$
\begin{aligned}
\left(a_{1}, \ldots, a_{n}\right) & \notin s=\left(\left(e_{1} \times \cdots \times e_{m}\right)^{-1}(r)\right)^{\varepsilon} \\
& \Longrightarrow\left(a_{\varepsilon(1)}, \ldots, a_{\varepsilon(m)}\right) \notin\left(e_{1} \times \cdots \times e_{m}\right)^{-1}(r) \\
& \Longrightarrow\left(e_{1}\left(a_{\varepsilon(1)}\right), \ldots, e_{m}\left(a_{\varepsilon(m)}\right)\right) \notin r \\
& \Longrightarrow\left(u\left(e_{1} \circ \rho_{\varepsilon(1)}\right), \ldots, u\left(e_{m} \circ \rho_{\varepsilon(m)}\right)\right) \notin r .
\end{aligned}
$$

Thus $r \in \operatorname{Fail}_{\underline{s}}^{\mathcal{B}}(u)$. 
It is natural to ask when the failset $U_{s}:=F_{s} \cap \Omega$ is a minimal failset of $s$. We now address this question.

Theorem 6.9 (The Minimal Failset Theorem). Assume that $\Omega$ contains all nonextendable partial endomorphisms of $\underline{M}$, or more generally that

(P) $\Omega$ contains some subset $P$ such that a map $v: D(\underline{s}) \rightarrow M$ preserves every non-extendable partial endomorphism if and only if $v$ preserves the relations in $P$.

If $F_{s}$ is a failset of $s$ and $F_{s}$ contains no non-extendable partial endomorphisms of $\underline{M}$ (equivalently, $F_{s} \cap P=\varnothing$ ), then $U_{s}:=F_{s} \cap \Omega$ is a minimal failset of $s$ and is the unique minimal failset of $s$ which does not intersect $P$. If $\Omega$ contains all non-extendable partial endomorphisms of $\underline{M}$, then $U_{s}$ is the unique minimal failset of $s$ which contains no non-extendable partial endomorphisms of $\underline{M}$.

Proof. Let $V$ be a failset which contains $s$ but does not intersect $P$. If

$$
s=\left(\left(e_{1} \times \cdots \times e_{m}\right)^{-1}(r)\right)^{\varepsilon}
$$

for some map $\varepsilon$, non-extendable partial endomorphisms $e_{1}, \ldots, e_{m}$ and $r \in \Omega$, then $\left\{e_{1}, \ldots, e_{m}, r\right\} \vdash s$, whence $P \cup\{r\} \vdash s$. Since $V$ contains $s$ and does not intersect $P$, it follows that $r \in V$, as $r \in \Omega$. Hence $U_{s}:=F_{s} \cap \Omega \subseteq V$. The case where $s \in V \subseteq U_{s}$ shows that $U_{s}$ is a minimal failset of $s$, while the case in which $V$ is not assumed to be contained in $U_{s}$ shows that $U_{s}$ is the unique minimal failset of $s$ which does not intersect $P$.

It is an extremely simple matter to guarantee that $\Omega$ contains all non-extendable partial endomorphisms of $\underline{M}$ : just insist that $\mathbb{S}\left(\underline{M}^{2}\right) \subseteq \Omega$. The following lemma shows that by modifying our assumptions we can ensure that $F_{s}$ will contain no partial endomorphisms at all. In this lemma we assume that $s$ is not a trivial expansion of a unary relation rather than $s$ is not a trivial expansion of eq $(e, f)$ for some pair of partial endomorphisms $e$ and $f$, since every unary relation $s$ can be written $s=\operatorname{eq}\left(\operatorname{id}_{s}, \operatorname{id}_{M}\right)$.

Lemma 6.10. Assume that $\underline{s} \leqslant M^{n}$, and that $s$ is not a trivial expansion of a unary relation and is not the trivial expansion of the joint kernel of two partial endomorphisms of $\underline{M}$. Then $F_{s}$ contains no partial endomorphisms of $\underline{M}$.

Proof. Assume that $F_{s}$ contains a partial endomorphism $e$. Thus there exist a map $\varepsilon:\{1,2\} \rightarrow\{1, \ldots, n\}$ and non-extendable partial endomorphisms $e_{1}$ and $e_{2}$ such that

$$
\begin{aligned}
s & =\left(\left(e_{1} \times e_{2}\right)^{-1}(e)\right)^{\varepsilon} & & \\
& =\left(\left(e_{2} \cdot\left(e_{1} \cdot e\right)^{\smile}\right)^{\smile}\right)^{\varepsilon} & & \text { as }\left(e_{1} \times e_{2}\right)^{-1}(e)=\left(e_{2} \cdot\left(e_{1} \cdot e\right)^{\smile}\right)^{\smile} \\
& =\left(\left(\operatorname{ker}\left(e_{2}, e_{1} \cdot e\right)\right)^{\smile}\right)^{\varepsilon} & & \text { as } \operatorname{ker}(f, g)=f \cdot g^{\smile} \\
& =\operatorname{ker}\left(e_{1} \cdot e, e_{2}\right)^{\varepsilon} & & \text { as } \operatorname{ker}(f, g)^{\smile}=\operatorname{ker}(g, f) .
\end{aligned}
$$

If $\varepsilon$ is a constant map then $s$ is a trivial expansion of the unary relation eq $\left(e_{1} \cdot e, e_{2}\right)$. If $\varepsilon$ is non-constant then $s$ is a trivial expansion of $\operatorname{ker}\left(e_{1} \cdot e, e_{2}\right)$.

We can refine the description of $F_{s}$. 


\section{Theorem 6.11.}

(a) If an m-ary relation $r$ belongs to $F_{s}$, then $s$ can be obtained from $r$ by application of the action of non-extendable partial endomorphisms alternating with $m$ applications of permutation of subscripts followed by a single application of $m$-to-n subscript manipulation.

(b) Assume that $F_{s}$ is a failset of $s$ (within $\mathcal{B}$ ) and $F_{s}$ contains no non-extendable partial endomorphism of $\underline{M}$. Then a relation $r \in \mathcal{B}$ is in $F_{s}$ if and only if $s$ can be obtained from $r$ via a finite number of applications of

(i) the action of non-extendable partial endomorphisms of $\underline{M}$, and

(ii) subscript manipulation.

(c) Assume that $\Omega$ satisfies (P) and that $\Omega$ is closed under the action of nonextendable partial endomorphisms of $\underline{M}$. If $F_{s}$ is a failset of $s($ within $\mathcal{B})$ and $F_{s}$ contains no partial endomorphism of $\underline{M}$, then $\Omega \backslash U_{s}\left(=\Omega \backslash F_{s}\right)$ is closed under the action of each non-extendable partial endomorphism of $\underline{M}$.

Proof. Let $\sigma$ be the cycle ( $m \ldots 21)$. It is easily seen that, if $r$ is not unary, then

$$
e \cdot r=\left(e \times \operatorname{id}_{\underline{M}} \times \cdots \times \operatorname{id}_{\underline{M}}\right)^{-1}(r)
$$

and that

$$
\left(e_{1} \times \cdots \times e_{m}\right)^{-1}(r)=\left(e_{m} \cdot \ldots \cdot\left(e_{2} \cdot\left(e_{1} \cdot r\right)^{\sigma}\right)^{\sigma} \ldots\right)^{\sigma} .
$$

This proves (a) since the unary case is trivial.

Now consider (b). If $r \in F_{s}$, then (by (a)), since permutation of subscripts is an instance of subscript manipulation, $s$ can be obtained from $r$ via a combination of at most $m$ applications of the action of non-extendable partial endomorphisms, and $m+1$ applications of subscript manipulation. For the converse we must assume that $F_{s}$ is a failset of $s$ which contains no non-extendable partial endomorphisms. If $s$ can be obtained from $r$ via a combination of the action of non-extendable partial endomorphisms $e_{1}, e_{2}, \ldots, e_{\ell}$ and subscript manipulation, then $\left\{e_{1}, e_{2}, \ldots, e_{\ell}, r\right\} \vdash$ $s$. Hence, since the complement of $F_{s}$ (in $\mathcal{B}$ ) is entailment-closed, if $e_{1}, \ldots, e_{\ell}, r \notin$ $F_{s}$, then we must have $s \notin F_{s}$. This is impossible because $F_{s}$ is a failset of $s$. Since $e_{1}, e_{2}, \ldots, e_{\ell} \notin F_{s}$, we conclude that $r \in F_{s}$.

Finally, assume that $\Omega$ satisfies (P) and that it is closed under the action of the non-extendable partial endomorphisms. If $F_{s}$ is a failset of $s$ which contains no non-extendable partial endomorphisms of $\underline{M}$, then $U_{s} \cap P=\varnothing$, by the Minimal Failset Theorem. Assume that $r \in \Omega$ is not unary and that $e$ is a non-extendable partial endomorphism of $\underline{M}$ such that $e \cdot r \in U_{s}$. Since $P \vdash e$ and $\{e, r\} \vdash e \cdot r$, we have $P \cup\{r\} \vdash e \cdot r$. Since $F_{s}$ is a failset (in $\mathcal{B}$ ) which contains no non-extendable partial endomorphisms, $U_{s}:=F_{s} \cap \Omega$ is a failset (in $\Omega$ ) which does not intersect $P$. Thus $P \cup\{r\} \vdash e \cdot r$ and $e \cdot r \in U_{s}$ imply that $r \in U_{s}$. Hence $r \in \Omega \backslash U_{s}$ implies $e \cdot r \in \Omega \backslash U_{s}$, as $\Omega$ is closed under the action of $e$. If $r$ is unary, replace each occurrence of $e \cdot r$ in the above argument by $e^{-1}(r)$.

Since the majority of our examples are lattice-ordered, we shall state versions of Theorems 6.8 and 6.9 appropriate to such examples. Note that every unary algebraic relation satisfies $(\mathrm{H})$. We give explicit descriptions of the unary and binary relations in $F_{s}$ in the case that $s$ itself is unary or binary. One additional item of notation is useful. Given partial endomorphisms $e$ and $f$ of $\underline{M}$ and $r \subseteq M^{2}$, define

$$
(e \otimes f)^{-1}(r):=\{c \in M \mid c \in \operatorname{dom} e \cap \operatorname{dom} f \text { and }(e(c), f(c)) \in r\} .
$$


Theorem 6.12. Let $s$ be unary. A unary relation $r$ belongs to $F_{s}$ if and only if $s=e^{-1}(r)$ for some non-extendable partial endomorphism e of $\underline{M}$. A binary relation $r$ is in $F_{s}$ if and only if $s=(e \otimes f)^{-1}(r)$ for some non-extendable partial endomorphisms $e$ and $f$ of $\underline{M}$.

Theorem 6.13. Let $s$ be binary. A unary relation $r$ belongs to $F_{s}$ if and only if $s=e^{-1}(r) \times M$ or $s=M \times e^{-1}(r)$ for some non-extendable partial endomorphism e of $\underline{M}$. A binary relation $r$ belongs to $F_{s}$ if and only if

$$
s \in\left\{(e \otimes f)^{-1}(r) \times M, M \times(e \otimes f)^{-1}(r),(e \times f)^{-1}(r),(e \times f)^{-1}(r)^{\smile}\right\}
$$

for some non-extendable partial endomorphisms $e$ and $f$.

We now wish to apply these results in order to characterise certain absolutely unavoidable relations.

Theorem 6.14. Let $\Omega=\mathbb{S}(\underline{M})$ and let $s$ be unary.

(a) Assume that $\{s\}$ is a minimal failset in $\Omega$. Then

(i) $\underline{s}$ is a value of $\underline{M}$, and

(ii) if $\Omega$ satisfies $(\mathrm{P})$ then $\mathbb{S}(\underline{M}) \backslash\{s\}$ is closed under the action of $e$ (or, equivalently, $s=e^{-1}(r)$ implies $\left.s=r\right)$, for every non-extendable partial endomorphism e of $\underline{M}$.

(b) Conversely, if

(i) $\underline{s}$ is a value of $\underline{M}$,

(ii) $s$ is not the domain of a proper non-extendable partial endomorphism of $\underline{M}$

(iii) $s$ is not the equaliser of two non-extendable partial endomorphisms of $\underline{M}$, and

(iv) $\mathbb{S}(\underline{M}) \backslash\{s\}$ is closed under the action of each non-extendable partial endomorphism of $M$,

then $\{s\}$ is a (minimal) failset in $\Omega$.

Proof. Assume that $\{s\}$ is a (minimal) failset in $\Omega$. Then $\underline{s}$ is a value of $\underline{M}$ since every failset contains a value (by Lemma 6.2). Assume that $\Omega$ satisfies $(\mathrm{P})$ and that $s \notin P$. Let $e$ be a non-extendable partial endomorphism of $\underline{M}$. Then $s=e^{-1}(r)$ implies that $\{e, r\} \vdash s$ and consequently $P \cup\{r\} \vdash s$. Since $\{s\}$ is a failset in $\Omega$ and $P \cup\{r\} \subseteq \Omega$, with $P \cap\{s\}=\varnothing$, we conclude that $r \in\{s\}$, so that $r=s$.

Now assume (i)-(iv) of (b). By (i)-(iii) and Theorem 6.8, the set $U_{s}:=F_{s} \cap \Omega$ is a failset of $s$ in $\Omega$. Let $r \in U_{s}$. Then $r$ is unary and by 6.12 there is a nonextendable partial endomorphism $e$ of $\underline{M}$ with $s=e^{-1}(r)$. Thus (iv) gives $r=s$, whence $\{s\}=U_{s}$ is a failset in $\Omega$. Hence (b) holds.

It is uncommon for $\mathbb{S}(\underline{M})$ to yield a duality on $\mathcal{A}$. As was pointed out in [3], Example 2.8, one important special case where this occurs is that in which $\underline{M}$ is semi-primal. A finite algebra $\underline{M}$ is semi-primal if the variety generated by $\underline{M}$ is both congruence-distributive and congruence-permutable, every subalgebra of $\underline{M}$ is simple and if $\varphi: \underline{N} \rightarrow \underline{N}^{\prime}$ is an isomorphism between non-trivial subalgebras, then $\varphi=\operatorname{id}_{\underline{N}}$.

Example 6.15. If $\underline{M}$ is a semi-primal algebra then $\mathbb{S}(\underline{M})$ yields a duality on $\mathcal{A}$, and a non-trivial subalgebra of $\underline{M}$ is absolutely unavoidable in $\mathbb{S}(\underline{M})$ if and only if it is a value of $\underline{M}$, or equivalently it is meet-irreducible in $\mathbb{S}(\underline{M})$. 
Proof. It follows from the definition of semi-primality that a non-extendable partial endomorphism is either the total map $\mathrm{id}_{\underline{M}}$, a total constant map onto a one-element subalgebra, or is the trivial isomorphism between two distinct one-element subalgebras. Thus if $s$ is a proper non-trivial subalgebra of $\underline{M}$, then $s$ is not the domain of a proper non-extendable partial endomorphism and is not the equaliser of two non-extendable partial endomorphisms. Moreover, if $e=\operatorname{id}_{M}$ or $e$ is a constant endomorphism and if $e^{-1}(r)$ is defined (that is, im $e \cap r \neq \varnothing$ ), then $e^{-1}(r) \in\{r, M\}$. Thus $\mathbb{S}(\underline{M}) \backslash\{s\}$ is closed under the action of $e$. Note that $\Omega$ satisfies (P): simply choose $P$ to be the set of all one-element subalgebras of $M$. It follows at once from the previous theorem that a non-trivial subalgebra $s$ of $\underline{M}$ is absolutely unavoidable in $\Omega$ if and only if $\underline{s}$ is a value of $\underline{M}$.

It follows that if $\underline{M}$ is a semi-primal algebra with no one-element subalgebras, then there is a unique optimal duality (within $\mathbb{S}(\underline{M})$ ) which is obtained by defining $R$ to be the set of values of $\underline{M}$.

We now move to the more usual situation where $\mathbb{S}\left(\underline{M}^{2}\right)$ is known to yield a duality. This occurs, for example, when $\underline{M}$ has a majority term, and in particular when $\underline{M}$ has an underlying lattice structure. Since we wish to use relations of minimum arity whenever possible, we shall consider both $\Omega=\mathbb{S}\left(\underline{M}^{2}\right)$ and $\Omega=$ $\mathbb{S}(\underline{M}) \cup \mathbb{S}\left(\underline{M}^{2}\right)$. As far as absolute unavoidability is concerned, $\mathbb{S}(\underline{M})$ will play no role, as it is easily seen that a unary relation is never absolutely unavoidable within $\mathbb{S}(\underline{M}) \cup \mathbb{S}\left(\underline{M}^{2}\right)$.

\section{Lemma 6.16.}

(a) A unary relation is never absolutely unavoidable within $\mathbb{S}(\underline{M}) \cup \mathbb{S}\left(\underline{M}^{2}\right)$.

(b) A binary relation of the form $s \times M$ or $M \times s$ for $s \subseteq M$ is neither absolutely unavoidable in $\mathbb{S}\left(M^{2}\right)$ nor absolutely unavoidable in $\mathbb{S}(\underline{M}) \cup \mathbb{S}\left(\underline{M}^{2}\right)$.

Proof. (b) Call the given relation $t$. In both cases $t$ is entailed by $\mathrm{id}_{\underline{s}}$, whence $\mathrm{id}_{\underline{s}} \in \Phi_{t}$ and consequently any minimal failset of $t$ contains $\mathrm{id}_{\underline{s}}$. Thus $t$ is not absolutely unavoidable.

In order to apply the Failset Theorem to show that $F_{s} \cap \Omega$ is a failset of $s$, we need to know that $s$ is not the joint kernel of two non-extendable partial endomorphisms. If $e$ is a partial endomorphism, then (the graph of) $e$ equals $\operatorname{ker}\left(e, \mathrm{id}_{\underline{M}}\right)$. Thus we shall assume that $s$ is not a non-extendable partial endomorphism. Although we have examples of endomorphisms which are absolutely unavoidable in $\mathbb{S}\left(\underline{M}^{2}\right)$, we lack a complete description of how this case can occur.

Theorem 6.17. Let $s \in \mathbb{S}(\underline{M}) \cup \mathbb{S}\left(\underline{M}^{2}\right)$. The conditions below are related as fol-

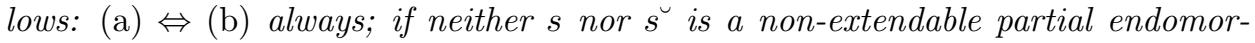
phism of $M$, then (a) $\Leftrightarrow(\mathrm{b}) \Rightarrow(\mathrm{c})$; if moreover $s$ is not the joint kernel of two non-extendable partial endomorphisms of $\underline{M}$ and s satisfies $(\mathrm{H})$, then (a) $\Leftrightarrow$ (b) $\Leftrightarrow(\mathrm{c})$.

(a) Either $\{s\}$ or $\{s, s\}$ is a minimal failset in $\mathbb{S}(\underline{M}) \cup \mathbb{S}\left(\underline{M}^{2}\right)$;

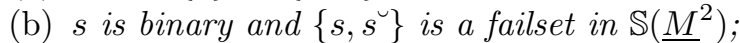

(c) $s$ is a value of $\underline{M}^{2}$ and is not a product of $M$ with a subalgebra of $\underline{M}$, and $\mathbb{S}\left(\underline{M}^{2}\right) \backslash\{s, s \triangleleft$ is closed under the action of the non-extendable partial endomorphisms of $\underline{M}$. 
Proof. The equivalence of (a) and (b) follows from the previous lemma. Assume that $s$ is binary and that $\left\{s, s^{\smile}\right\}$ is a minimal failset in $\mathbb{S}\left(\underline{M}^{2}\right)$. The subalgebra $\underline{s}$ is a value of $\underline{M}^{2}$, by 6.2 , and is not a product of $\underline{M}$ with a subalgebra of $\underline{M}$, by the previous lemma. Assume that $e \cdot r \in\{s, s \breve{s}\}$ for some binary relation $r$ and some non-extendable partial endomorphism $e$. Thus $\{e, r\} \vdash s$ and, since $\{s, s\}$ is a failset, it follows that either $e \in\{s, s\}$ or $r \in\{s, s u$. If neither $s$ nor $s \breve{\text { is a }}$

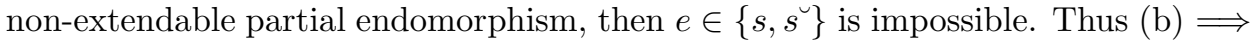
(c).

Finally assume that $s$ is not the joint kernel of two non-extendable partial endomorphisms, that $s$ satisfies (H), and that (c) holds. By the Failset Theorem, $F_{s}$ is a failset of $s$. Since $s$ is not the product of $M$ with some subalgebra of $\underline{M}$, by 6.13 we have that $r \in F_{s} \cap \mathbb{S}\left(\underline{M}^{2}\right)$ if and only if $r$ is a binary relation such that $s=(e \times f)^{-1}(r)$ or $s=\left((e \times f)^{-1}(r)\right)^{\triangleleft}$ for some non-extendable partial endomorphisms $e$ and $f$ of $\underline{M}$. Since $(e \times f)^{-1}(r)=\left(f \cdot(e \cdot r)^{\iota}\right)^{\triangleleft}$, a simple calculation shows

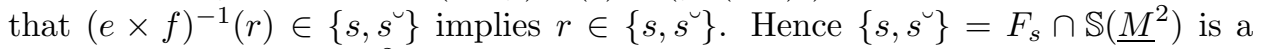
(minimal) failset in $\mathbb{S}\left(\underline{M}^{2}\right)$.

\section{Pseudocomplemented Distributive Lattices Revisited}

In [9] we applied the test algebra technique to derive optimal dualities for the finitely generated varieties $\mathbf{B}_{n}$ of pseudocomplemented distributive lattices. To do this we made heavy use of the machinery of distributive lattice duality. Here we show how these results follow from the general theory developed above. Our presentation in this section is rather condensed, and many of our algebraic assertions may be most easily confirmed by interpreting the subalgebras in dual form, as in [8], [9]. This is particularly true of the proofs of the later theorems.

Recall that $\mathbf{B}_{\omega}$ is the class of algebras $\left(A ; \vee, \wedge,{ }^{*}, 0,1\right)$ of type $(2,2,1,0,0)$ such that $(A ; \vee, \wedge, 0,1)$ is a bounded distributive lattice and $a^{*}$ (the pseudocomplement of $a$ ) is given by

$$
a^{*}=\max \{b \in A \mid a \wedge b=0\} .
$$

The lattice of subvarieties of $\mathbf{B}_{\omega}$ is an $\omega+1$ chain

$$
\mathbf{B}_{-1} \subset \mathbf{B}_{0} \subset \mathbf{B}_{1} \subset \cdots \subset \mathbf{B}_{n} \subset \cdots \subset \mathbf{B}_{\omega},
$$

where $\mathbf{B}_{-1}$ is the trivial variety, $\mathbf{B}_{0}$ is the variety of Boolean algebras and $\mathbf{B}_{1}$ is the variety of Stone algebras. For $n \geqslant 0$, we have $\mathbf{B}_{n}=\mathbb{I S P}\left(\underline{P}_{n}\right)$, where the algebra $\underline{P}_{n}$ is obtained by adjoining a new top to the $n$-atom Boolean lattice. We denote the coatom in $\underline{P}_{n}$ by $d$. Every subalgebra of $\underline{P}_{n}$ is isomorphic to $\underline{P}_{m}$ for some $m$ with $0 \leqslant m \leqslant n$.

For the remainder of our discussion, fix $n \geqslant 1$. The distributive lattice homomorphism $\alpha: \underline{P}_{n} \rightarrow\{0,1\}$, which sends 1 in $\underline{P}_{n}$ to 1 and all other elements of $\underline{P}_{n}$ to 0 , plays a vital role in [8]. Indeed, the general piggyback theory from [12], [13] shows that the endomorphisms of $\underline{P}_{n}$ along with the set $T$ of subalgebras of $\underline{P}_{n}^{2}$ which are maximal in

$$
\alpha^{-1}(\leqslant):=\left\{(a, b) \in \underline{P}_{n}^{2} \mid \alpha(a) \leqslant \alpha(b)\right\}
$$

yield a duality on $\mathbf{B}_{n}$. These subalgebras were described, dually and algebraically, in [8] (less precise information is obtained by purely algebraic means in [13]). Here 
it is most convenient to use the fact that every $s \in T$ is of the form

$$
E(g):=\{(c, g(c)) \mid c \in \downarrow d\} \cup\{(c, 1) \mid g(c)=d\} \cup\{(1,1)\},
$$

where $g: \downarrow d \rightarrow \downarrow d$ is a Boolean endomorphism. Note in particular that if $s \in T$ then $(d, d),(d, 1)$ and $(1,1) \in s$ but $(1, d) \notin s$, and that $|s| \geqslant 2^{n}+2$. In fact, as the following lemma shows, $s$ is a value at $(1, d)$.

Lemma 7.1. A subalgebra $\underline{s}$ of $\underline{P}_{n}^{2}$ is a value at $(1, d)$ if and only if $s=P_{n} \times\{0,1\}$ or $s$ is maximal in $\alpha^{-1}(\leqslant)$.

Proof. Assume that $s \in T$ and assume that $s \varsubsetneqq r \subseteq \underline{P}_{n}^{2}$. Then $r \nsubseteq \alpha^{-1}(\leqslant)$, whence there exists $(b, c) \in r$ such that $(\alpha(b), \alpha(c))=(1,0)$. Thus $(1, c) \in r$. Also $c<1$, so $(1, d)=(1, c) \vee(d, d) \in r$ (since $(d, d) \in s)$. Thus any $s \in T$ is indeed a value at $(1, d)$. Since $\{0,1\}$ is a value of $\underline{P}_{n}$ at $d$, it follows that $\underline{P}_{n} \times\{0,1\}$ is also a value at $(1, d)$.

Conversely, assume that $\underline{s}$ is a value of $\underline{P}_{n}^{2}$ at $(1, d)$ and that $s$ is not $\underline{P}_{n} \times\{0,1\}$. If $\pi_{2}(s)=\{0,1\}$ then, by the fact that $s$ is maximal with respect to not containing $(1, d)$, we would have $s=\underline{P}_{n} \times\{0,1\}$, contrary to hypothesis. Any subalgebra of $\underline{P}_{n}$ other than $\{0,1\}$ contains $d$. Thus there exists $b<1$ such that $(b, d) \in s$. If $s \nsubseteq$ $\alpha^{-1}(\leqslant)$, then there exists $c \leqslant d$ such that $(1, c) \in s$. Then $(1, d)=(1, c) \vee(b, d) \in s$, which is impossible. Thus $s \subseteq \alpha^{-1}(\leqslant)$. No subalgebra of $\alpha^{-1}(\leqslant)$ contains $(1, d)$, so the assumption that $\underline{s}$ is a value forces $s$ to be maximal in $\alpha^{-1}(\leqslant)$.

We shall need the following elementary facts, which we collect together for future reference.

\section{Lemma 7.2.}

(a) Let e be a partial endomorphism of $\underline{P}_{n}$. Then either

(i) $e$ is one-to-one, or

(ii) e has codomain $\{0,1\}$ and $e \uparrow_{(\downarrow d \cap \text { dome } e)}$ is a Boolean homomorphism.

(b) Assume that $r, s \subseteq \underline{P}_{n}^{2}$ with $s \in T$ and let $e$ and $f$ be partial endomorphisms of $\underline{P}_{n}$ such that $s=(e \times f)^{-1}(r)$. Then e and $f$ are one-to-one.

Proof. The first part is established algebraically in [13] and dually in [8].

Assume that $s=(e \times f)^{-1}(r)$. Since $(d, d) \in s$, we have $d \in \operatorname{dom} e \cap \operatorname{dom} f$. Now $b \in \operatorname{dom} f$ and $f(b)=0$ implies $(e(0), f(b))=(0,0) \in r$, so $(0, b) \in s$. This happens for $s \in T$ only if $b=0$ (since $s=E(g)$ as described in (*) above), which implies by (a) that $f$ is one-to-one, whence in particular $f(d)=d$. If $e$ is not one-to-one then $e(d)=e(1)=1$, whence

$$
\begin{aligned}
(d, d) \in s & \Longrightarrow(e(d), f(d)) \in r \\
& \Longrightarrow(e(1), f(d)) \in r \\
& \Longrightarrow(1, d) \in s,
\end{aligned}
$$

which is false. Thus $e$ is indeed one-to-one.

We now apply the Minimal Failset Theorem. See 6.9 and the paragraph before 6.8 for the definition of $U_{s}$.

Proposition 7.3. Let $\underline{s} \leqslant \underline{P}_{n}^{2}$ be maximal in $\alpha^{-1}(\leqslant)$ and let $r \in \Omega:=\mathbb{S}\left(\underline{P}_{n}\right) \cup$ $\mathbb{S}\left(\underline{P}_{n}^{2}\right)$. Then $r \in U_{s}$ if and only if $r$ is binary and there exist non-extendable partial 
endomorphisms $e$ and $f$ such that

$$
(e \times f)^{-1}(r)=\left(f \cdot(e \cdot r)^{\smile}\right)^{\smile} \in\left\{s, s^{\smile}\right\} .
$$

Proof. We apply the Minimal Failset Theorem with $\Omega=\mathbb{S}\left(\underline{P}_{n}\right) \cup \mathbb{S}\left(\underline{P}_{n}^{2}\right)$. In order to do so, we need to rule out the possibility that $s$ satisfies (D), (E) or (K). Note that certainly $s$ is not a product, as $(1,1)$ is join-irreducible in $\underline{s}$. Now suppose it were the case that $s=\operatorname{ker}(e, f)=e \cdot f^{\lrcorner}$for partial endomorphisms $e$ and $f$. By Lemma 7.2(b) (with $f=$ id and $r=f^{\sqcup}$ ) we have that $e$ is one-to-one. Further, if $(b, a) \in \operatorname{ker}(e, f)$ and $(c, a) \in \operatorname{ker}(e, f)$, then $e(b)=f(a)=e(c)$, whence we deduce that $b=c$. This implies that the converse of $s$ is the graph of a partial endomorphism. However, because $(d, 1)$ belongs to $s$, this is not the case. We conclude that $s$ cannot be the joint kernel of partial endomorphisms.

We may now apply the Failset Theorem, the Minimal Failset Theorem and Lemma 6.10 to obtain that $U_{s}$ is the unique minimal failset of $s$ which contains no non-extendable partial endomorphisms. Moreover, by Theorem $6.13, U_{s}$ contains no unary relations (since $s$ is not a product) and a binary relation $r$ is in $U_{s}$ if and only if there are non-extendable partial endomorphisms $e$ and $f$ such that $(e \times f)^{-1}(r) \in\left\{s, s^{\iota}\right\}$.

The elements of $T$ fall into $n$ equivalence classes under lattice isomorphism (called shapes in [9]). The principal results of [9] assert that

(i) a generating set for End $\underline{P}_{n}$ together with one maximal subalgebra of $\alpha^{-1}(\leqslant)$ of each shape yields a duality on $\mathbf{B}_{n}$, and

(ii) any subset of End $\underline{P}_{n} \cup T$ yielding a duality must contain at least one relation of each shape.

To show how these results may be obtained from our general theory, and indeed sharpened, we must first investigate how isomorphisms act on the sets $U_{s}$ for $s \in T$.

Proposition 7.4. Let $\Omega=\mathbb{S}\left(\underline{P}_{n}\right) \cup \mathbb{S}\left(\underline{P}_{n}^{2}\right)$, let $\underline{s} \leqslant \underline{P}_{n}^{2}$ be maximal in $\alpha^{-1}(\leqslant)$ and let $r \in U_{s}$. Then $r$ is binary and $\underline{r} \cong \underline{s}$.

Proof. By Proposition 7.3 it suffices to show that if $s=e \cdot r$ for some non-extendable partial endomorphism $e$, then $\underline{r} \cong \underline{s}$. Assume that $s=e \cdot r$. We claim that $e \times$ id: $s \rightarrow \underline{P}_{n}^{2}$ is one-to-one with image $r$. That $e \times$ id is one-to-one follows at once from Lemma 7.2(b). Next note that $s=e \cdot r=(e \times \mathrm{id})^{-1}(r)$ implies that $(e \times \mathrm{id})(s) \subseteq r$. It follows immediately from the algebraic description of $s$ given in $(*)$ above that $\pi_{1}(s)=\underline{P}_{n}$, and since $s \subseteq \operatorname{dom} e \times \underline{P}_{n}$ we must have dom $e=\underline{P}_{n}$. But then, because $e$ is one-to-one, $e$ is an automorphism of $\underline{P}_{n}$. It follows that $r=e^{-1} \cdot e \cdot r=e^{-1} \cdot s$, whence $e^{-1} \times \mathrm{id}: r \rightarrow s$ is a well-defined inverse to $e \times$ id $: s \rightarrow r$. Hence $\underline{r} \cong \underline{s}$, as required.

Proposition 7.5. Let $\Omega=\mathbb{S}\left(\underline{P}_{n}\right) \cup \mathbb{S}\left(\underline{P}_{n}^{2}\right)$, let $s \in T$, and let $r \in U_{s}$ with $\underline{r} \cong \underline{s}$. Then $r$ is binary, and either $r$ or $r^{\smile}$ belongs to $T$.

Proof. Since $|r|=|s| \geqslant 2^{n}+2$, the relation $r$ cannot be unary. We may assume without loss of generality that $(e \times f)^{-1}(r)=s$. By Lemma 7.2, both $e$ and $f$ are one-to-one. Then $(1, d)$ is a fixpoint of $e \times f$, and because $(1, d) \notin s$ we have $(1, d) \notin r$. Suppose $r \subseteq t \subseteq \underline{P}_{n}^{2}$ with $(1, d) \notin t$. Then $s \subseteq(e \times f)^{-1}(t)$, and $(1, d) \notin(e \times f)^{-1}(t)$, again because $(1, d)$ is a fixpoint of $e \times f$. Thus, because $s$ is a value at $(1, d)$, we have $s=(e \times f)^{-1}(t)=\left(f \cdot(e \cdot t)^{\smile}\right)^{\smile}$. Therefore $|r|=|s|=|t|$, 
whence $r=t$, so that $r$ is a value at $(1, d)$. Since $r \cong s$ it is impossible that $r=\underline{P}_{n}^{2} \times\{0,1\}$, and, by Proposition 7.1, we have $r \in \bar{T}$.

In the following proposition we shall, for clarity, explicitly indicate the domains of restricted projection maps (by superscripting).

Proposition 7.6. Let $\Omega=\mathbb{S}\left(\underline{P}_{n}\right) \cup \mathbb{S}\left(\underline{P}_{n}^{2}\right)$. Assume that $r, s \subseteq \underline{P}_{n}^{2}$ and $s \in T$. Let $\gamma: \underline{s} \rightarrow \underline{r}$ be an isomorphism. Then there is a map $u: D(\underline{s}) \rightarrow P_{n}$ such that $r \in \operatorname{Fail}_{\underline{s}}(u)=U_{s}$, and this is witnessed by $x_{1}, x_{2}$ defined by $x_{i}:=\rho_{i}^{r} \circ \gamma$.

Proof. We have already shown (see the proof of 7.3) that $s$ does not satisfy (D), (E) or $(\mathrm{K})$ of 6.8 . Thus, by 6.7 and $7.1, U_{s}=$ Fail $_{\underline{s}}(u)$, where, for all $x \in D(\underline{s})$, we define $u(x)=1$ if $x=e \circ \rho_{1}^{s}$ and $u(x)=e(d)$ if $x=e \circ \rho_{2}^{s}$, for some nonextendable partial endomorphism $e$. Let $\gamma: \underline{s} \rightarrow \underline{r}$ be an isomorphism and define $x_{1}, x_{2}$ as in the statement of the proposition. Note that $x_{1}, x_{2} \in D(\underline{s})$, and choose non-extendable partial endomorphisms $e_{1}, e_{2}$ and a map $\varepsilon:\{1,2\} \rightarrow\{1,2\}$ such that $x_{i}=e_{i} \circ \rho_{\varepsilon(i)}^{s}$. If $\varepsilon$ is the constant function onto 1 , then $\gamma$ depends only on the first coordinate, whence $\gamma(d, d)=\gamma(d, 1)$. Similarly, if $\varepsilon$ is the constant map onto 2 , then we obtain the contradiction $\gamma(d, 1)=\gamma(1,1)$. Now assume that $\varepsilon=\mathrm{id}_{\{1,2\}}$. Thus for any $\left(c_{1}, c_{2}\right) \in s$, we have $\gamma\left(c_{1}, c_{2}\right)=\left(e_{1}\left(c_{1}\right), e_{2}\left(c_{2}\right)\right)$. If $e_{1}$ is not one-to-one, then $e_{1}(d)=e_{1}(1)$, whence

$$
\gamma(d, 1)=\left(e_{1}(d), e_{2}(1)\right)=(1,1)=\left(e_{1}(1), e_{2}(1)\right)=\gamma(1,1)
$$

contradicting the fact that $\gamma$ is an isomorphism. In the same way, if $e_{2}$ is not one-to-one,

$$
\gamma(d, d)=\left(e_{1}(d), e_{2}(d)\right)=\left(e_{1}(d), e_{2}(1)\right)=\gamma(d, 1),
$$

again a contradiction. Thus both $e_{1}$ and $e_{2}$ are one-to-one. Hence

$$
(d, 1)=\left(e_{1}(d), e_{2}(1)\right)=\gamma(d, 1) \in r .
$$

Note that if both $(d, 1) \in r$ and $(1, d) \in r$, then we would have $(1,1)$ join-reducible in $\underline{r}$, which contradicts the join-irreducibility of $(1,1)$ in $\underline{s}$. Therefore $(1, d) \notin r$. We conclude that $x_{1}, x_{2}$ do witness $r \in \operatorname{Fail}_{\underline{s}}(u)$, since $\left(x_{1}, x_{2}\right) \in r_{D(\underline{s})}$ by the $r$-on- $s$ Lemma, 2.2, while

$$
\left(u\left(x_{1}\right), u\left(x_{2}\right)\right)=\left(1, e_{2}(d)\right)=(1, d) \notin r .
$$

The case that $\varepsilon=\left(\begin{array}{ll}1 & 2\end{array}\right)$ is a simple variant of the case that $\varepsilon=\operatorname{id}_{\{1,2\}}$.

We sum up and refine our results in the following theorem.

Theorem 7.7. Let $\Omega=\mathbb{S}\left(\underline{P}_{n}\right) \cup \mathbb{S}\left(\underline{P}_{n}^{2}\right)$ and let $\underline{s} \leqslant \underline{P}_{n}^{2}$ be maximal in $\alpha^{-1}(\leqslant)$.

(a) Every relation in $U_{s}$ is binary.

(b) The following are equivalent for $r \subseteq P_{n}^{2}$ :

(i) $r \in U_{s}$;

(ii) $\underline{r} \cong \underline{s}$;

(iii) End $\underline{P}_{n} \cup\{r\} \vdash s$;

(iv) End $\underline{P}_{n} \cup\{r\} \vdash s$ and End $\underline{P}_{n} \cup\{s\} \vdash r$;

(v) $U_{s}=U_{r}$.

(c) $U_{s}$ equals $\left\{r \subseteq P_{n}^{2} \mid \underline{r} \cong \underline{s}\right\}$ and is a (globally) minimal failset both in $\mathbb{S}\left(\underline{P}_{n}^{2}\right)$ and in $\mathbb{S}\left(\underline{P}_{n}\right) \cup \mathbb{S}\left(\underline{P}_{n}^{2}\right)$. 
Proof. Part (a) follows immediately from Proposition 7.3. Now consider (b). The equivalence of (i) and (ii) comes from Propositions 7.4 and 7.6. Since $U_{s}$ contains no partial endomorphisms, End $\underline{P}_{n} \cup\{r\} \vdash s$ implies that $r \in U_{s}$ since $s \in U_{s}$. Thus (iii) implies (i), while (i) $\Rightarrow$ (iii) is an immediate consequence of the fact that $r \in U_{s}$ implies that $s$ may be constructed as in Proposition 7.3. Now assume

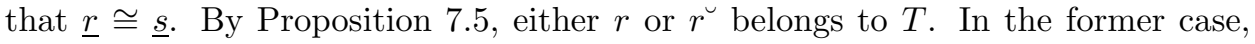
Proposition 7.6 (with $r$ and $s$ interchanged) implies that $s \in U_{r}$. In the latter case,

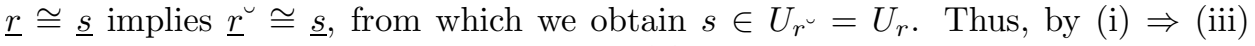
with $s$ replaced by $r$, we have End $\underline{P}_{n} \cup\{s\} \vdash r$, whence (ii) implies (iv). Since $r \in U_{s} \Rightarrow \underline{r} \cong \underline{s} \Rightarrow s \in U_{r}$, it follows at once that (i) is equivalent to (v). Finally, since $U_{r}=U_{s}$ for all $r \in U_{s}$, the set $U_{s}$ is a minimal failset of each of its members, whence $U_{s}$ is a (globally) minimal failset by Proposition 3.14. Thus (c) holds.

This gives a new and purely algebraic proof of the main optimal duality theorem of our previous paper.

Theorem 7.8 (cf. [9], Theorem 5.1). Let $S$ be a transversal of the isomorphism classes of $T$, the set of maximal subalgebras of $\alpha^{-1}(\leqslant)$. Then End $\underline{P}_{n} \cup S \vdash T$, and End $\underline{P}_{n} \cup S$ yields a duality on $\mathbf{B}_{n}$.

The theory presented in this paper gives little information about minimal failsets which contain endomorphisms. Nevertheless we can be quite explicit about such failsets for the varieties $\mathbf{B}_{n}$. First we see that Theorem 6.5 handles the minimal failsets of endomorphisms which are not automorphisms.

Proposition 7.9. Let $\Omega=\mathbb{S}\left(\underline{P}_{n}\right) \cup \mathbb{S}\left(\underline{P}_{n}^{2}\right)$ and $N=\{0,1\}$. The set $\Phi_{N}$ is a globally minimal failset, containing the following relations (and no others):

(a) the unary relation $N$,

(b) graphs of partial endomorphisms with codomain $\{0,1\}$ and their converses, and

(c) relations $r$ such that $\underline{r} \cong \underline{P}_{m} \times \underline{N}(0 \leqslant m \leqslant n)$ and their converses.

If $\Omega$ is chosen to be $\mathbb{S}\left(\underline{P}_{n}^{2}\right)$, then $\Phi_{\Delta_{N}}$ is a globally minimal failset and contains precisely the relations described in (b) and (c) above.

Proof. The subalgebra $\underline{N}$ is a value at $d$ and at no other point, and $|D(\underline{N})|=1$. Thus Theorem 6.5 applies.

By Lemma 7.2 any endomorphism $e$ which is not an automorphism is such that graphe $\cap \Delta_{\underline{P}_{n}}=\Delta_{N}$, and so belongs to $\Phi_{N}$. Also $(d, d) \in r$ implies $r \notin \Phi_{N}$. This means that $r \in \Phi_{N}$ only if either $\operatorname{im} \rho_{1}=\{0,1\}$ or $\operatorname{im} \rho_{2}=\{0,1\}$. Assume $\pi_{2}(r)=\{0,1\}$. The image $\pi_{1}(r)$ is a subalgebra of $\underline{P}_{n}$ and hence of the form $\underline{P}_{m}$ for some $m \leqslant n$. Assume first $(0,1) \in r$. We claim that $r=P_{m} \times\{0,1\}$ in this case. Certainly $r \subseteq P_{m} \times\{0,1\}$. For $a \in \pi_{1}(r)$, we have $(a, 0) \in r$ or $(a, 1) \in r$. In the former case, $(a, 1)=(a, 0) \vee(0,1) \in r$, and in the latter case, $(a, 0)=(a, 1) \wedge\left(0^{*}, 1^{*}\right) \in r$. This proves the claim. Now assume $(0,1) \notin r$, whence also $(1,0) \notin r$. Suppose that there exist $a, b, c$ such that $(a, b) \in r$ and $(a, c) \in r$. If $b \neq c$, we have, without loss of generality, $b=0$ and $c=1$, and so $a \neq 0$ or 1 . If $a$ were equal to $d$ we would have $(0,1)=\left(a^{*}, 0^{*}\right) \in r$, contrary to hypothesis. Consequently, $0<a<d$ and $(0,1)=\left((a, 0) \vee\left(a^{*}, 1^{*}\right)\right)^{*} \in r$, which is again a contradiction. Therefore $r$ is the graph of a partial endomorphism.

Finally, we show that $\Phi_{N}$ is globally minimal. Certainly $\Phi_{N}$ is the unique locally minimal failset of $N$, by Theorem 6.5. Suppose, for a contradiction, that there 
exists a globally minimal failset $U$ contained in $\Phi_{N}$ and not containing $N$. Assume $U$ contains a relation $s$ of type (c), say $s=N \times P_{m}$, and let $U=$ Fail $_{s}(u)$. Then $u$ preserves $N$ and $P_{m}$ (neither of which lies in $U$ ) and hence preserves their product, contrary to hypothesis. Hence $U$ contains no relation of type (c). Thus $U$ consists solely of relations of type (b). Assume $e \in U$, where $e$ is a partial endomorphism with codomain $\{0,1\}$. There is a Boolean endomorphism $g: \downarrow d \rightarrow \downarrow d$ such that $E(g) \cap(\operatorname{dom} e \times N)$ is the graph of $e$. (To construct such a $g$ let $f \in \operatorname{End} \underline{P}_{n}$ be an extension of $e$, with codomain $\{0,1\}$. Define $g(a)=0$ if $f(a)=0$, and $g(a)=d$ if $f(a)=1$.) Since $E(g)$, dome and $N$ are not in $U$, it follows that $e \notin U$; a contradiction.

The claims concerning the binary-only case follow immediately.

We must now consider failsets containing automorphisms. Here we have no general theory to call on, but can proceed in an ad hoc fashion quite simply. For any $s \in$ End $P_{n}$, there is a natural bijection between $D(\underline{s})$ and $D\left(\underline{P}_{n}\right)=$ End $\underline{P}_{n}$, given by $D\left(\rho_{1}\right)$, under which $\rho_{1}$ and $\rho_{2}$ in $D(\underline{s})$ correspond to id and $s$, and the endomorphisms act by composition. For a given $v: D(\underline{s}) \rightarrow P_{n}$, we have $\operatorname{Fail}_{\underline{s}}(v)=$ Fail $_{\underline{P}_{n}}(u)$, where $u=v \circ D\left(\rho_{1}\right)$ (using $2.4(\mathrm{~b})$ ). We shall henceforth identify $D(\underline{s})$ with End $\underline{P}_{n}$.

Recall that Aut $\underline{P}_{n}$ is isomorphic to the permutation group $S_{n}$. Among the maximal subalgebras of $\alpha^{-1}(\leqslant)$ we have the following, indexed by the members $f$ of Aut $\underline{P}_{n}$ :

$$
f^{\leqslant}:=\operatorname{graph}(f) \cup\{(d, 1)\} .
$$

The notation here is chosen to acknowledge the fact that $f \leqslant=f \cdot \preccurlyeq$, where $\preccurlyeq$ is the partial order on $P_{n}$ whose associated strict order has $d<1$ as its only comparability.

Proposition 7.10. Let $\Omega=\mathbb{S}\left(\underline{P}_{n}\right) \cup \mathbb{S}\left(\underline{P}_{n}^{2}\right)$. Let $H$ be a subgroup of Aut $\underline{P}_{n}$ and denote its complement in Aut $\underline{P}_{n}$ by $K$. Then

$$
W_{H}:=K \cup\left\{f^{\leqslant} \mid f \in K\right\} \cup\left\{\left(f^{\leqslant}\right)^{\cup} \mid f \in K\right\}
$$

is a failset of each of its members.

Proof. Let $s \in K$. Then, as noted above, we may identify $D(\underline{s})$ with End $\underline{P}_{n}$, which consists of the automorphisms of $\underline{P}_{n}$ together with the $\{0,1\}$-valued endomorphisms. Define $u: D(\underline{s}) \rightarrow P_{n}$ by

$$
u(x):= \begin{cases}d & \text { if } x \in K, \\ 1 & \text { otherwise }\end{cases}
$$

Let $x \in K$. Then $u(x \circ$ id $)=u(x)=d$, while $x(u($ id $))=x(1)=1$, so that $u$ fails to preserve $x$ for each $x \in K$ (recall the identification of id $\in H$ with $\rho_{1}$ ). Now let $x \in H$. For any $y \in \operatorname{Aut} \underline{P}_{n}$, we have $x \circ y \in H$ if and only if $y \in H$, so that $u(x \circ y)=x(u(y))$. For $y \in$ End $\underline{P}_{n} \backslash$ Aut $\underline{P}_{n}$, we have $x \circ y \in$ End $\underline{P}_{n} \backslash$ Aut $\underline{P}_{n}$ too, so again $u(x \circ y)=x(u(y))$. Therefore $u$ preserves every element of $H$. If $x \in$ End $\underline{P}_{n} \backslash$ Aut $\underline{P}_{n}$, then $u(x \circ y)=1=x(u(y))$ for every $y \in$ End $\underline{P}_{n}$, whence $u$ preserves $x$.

Next consider $\underline{r} \leqslant \underline{P}_{n}^{2}$, and assume that $r$ is not contained in either $\alpha^{-1}(\leqslant)$ or $\alpha^{-1}(\geqslant)$. Then there exist $b, c<1$ such that $(1, b) \in r$ and $(c, 1) \in r$. First assume that neither $b$ or $c$ is $0 . \quad\left(0, b^{*}\right) \in r$ and $\left(c^{*}, 0\right) \in r$. We deduce that $(1, d)=(1, b) \vee\left(0, b^{*}\right) \in r$ and similarly $(d, 1) \in r$. Then $(d, d)=(d, 1) \wedge(1, d) \in r$ 
and $(1,1) \in r$ automatically. Since $u$ maps into $\{d, 1\}$, the map $u$ preserves $r$. Now assume that $b$ nor $c$ is 0 . If we know that $(0,1) \in r$ then $(1,0) \in r$ too (and vice versa). Let $N_{i}=\pi_{i}(r)$. We claim that $r=N_{1} \times N_{2}$. Certainly $r \subseteq N_{1} \times N_{2}$. Take $(a, b) \in N_{1} \times N_{2}$. Then there exist $a_{1}, b_{1}$ such that $\left(a, b_{1}\right)$ and $\left(a_{1}, b\right)$ belong to $r$. But then $(a, 1)=\left(a, b_{1}\right) \vee(0,1) \in r$ and $(1, b) \in r$ similarly. Therefore $(a, b)=(a, 1) \wedge(1, b) \in r$. This proves our claim that $r$ is the product $N_{1} \times N_{2}$. Suppose $r$ is a product $N_{1} \times N_{2}$. We wish to show that on $D(\underline{s})=$ End $\underline{P}_{n}$ the map $u$ which sends all endomorphisms except those in $K$ to 1 must preserve $r$. The only problem that could arise is when $(d, d),(d, 1)$ or $(1, d)$ fails to belong to $r$, that is, when at least one of the factors of $r$ is $\{0,1\}$. We have $\left(x_{1}, x_{2}\right) \in r_{D(\underline{s})}$ if and only if $x_{1}$ maps into $N_{1}$ and $x_{2}$ maps into $N_{2}$. But $x_{i}$ is an automorphism if and only if $N_{i}=\underline{P}_{n}$, and so contains $d$. This means that $u$ does preserve $r$.

Now take $r \subseteq \alpha^{-1}(\leqslant)$ and assume that $r$ is not a subalgebra of $f \leqslant$. In this situation we have that $r$ is contained in a subalgebra $E(g)$ (as in (*) above), where $g$ is not an automorphism of $\downarrow d$. Hence $\pi_{2}(r)$ is a proper subset of $P_{n}$. Consider endomorphisms $x$ and $y$, where $y$ is an automorphism. Then $(x, y) \in r_{D(\underline{s})}$ would imply that $\pi_{2}$ maps onto $P_{n}$. Thus we can only have $(x, y) \in r_{D(\underline{s})}$ if $y=\bar{e}$, where $e$ is a $\{0,1\}$-valued endomorphism. This means that $(u(x), u(y))$ must be $(d, 1)$ or $(1,1)$, either of which is in $r$. Thus $u$ preserves $r$.

Consider the case that $r$ is $f \leqslant$. Then, for endomorphisms $x$ and $y$ of $\underline{P}_{n}$, we have $(x, y) \in r_{D(\underline{s})}$ if and only if $y=f \circ x$. Consequently,

$$
f \in \operatorname{Fail}_{\underline{P}_{n}}(u) \Longleftrightarrow f \leqslant \operatorname{Fail}_{\underline{P}_{n}}(u) \Longleftrightarrow\left(f^{\leqslant}\right)^{\smile} \in \operatorname{Fail}_{\underline{P}_{n}}(u) .
$$

Finally, assume that $r$ is a subalgebra properly contained in $f \leqslant$ (or its converse) and is not an automorphism. Then $r$ is either the graph of a proper partial endomorphism, or such a graph with $(d, 1)$ added (or $(1, d)$ added). It is only possible to have $(x, y) \in r_{D(s)}$ if $x, y$ are not automorphisms, since neither of the projections maps $r$ onto $P_{n}$. For such $x, y$ we have $(u(x), u(y)) \in r$, so $r$ is preserved by $u$.

When $r$ is unary, $x \in r_{D(\underline{s})}$ if and only if im $x \subseteq r$. When $r \neq \underline{P}_{n}$ this happens only if $x$ has codomain $\{0,1\}$, and so $u$ preserves $r$.

We have shown that $W_{H}=\operatorname{Fail}_{f}(u)$ for any $f \in K$. Now let $r=f \leqslant$, where $f \in K$. Then $f$ is a retract of $r$, so there exists $v: D(\underline{r}) \rightarrow P_{n}$ such that $\operatorname{Fail}_{f}(u)=$ Fail $_{\underline{r}}(v)$, by Lemma 2.4(b). It follows that $W_{H}$ is a failset of each of its members.

We are primarily interested in minimal failsets.

Theorem 7.11. The globally minimal failsets in $\Omega=\mathbb{S}\left(\underline{P}_{n}\right) \cup \mathbb{S}\left(\underline{P}_{n}^{2}\right)$ which have a non-empty intersection with Aut $\underline{P}_{n}$ are precisely the sets $W_{H}$, where $H$ ranges over the maximal proper subgroups of Aut $\underline{P}_{n} \cong S_{n}$.

Proof. Let $U$ be a globally minimal failset containing an automorphism. Let $K=$ $U \cap$ Aut $\underline{P}_{n}$, with $H$ denoting its complement in Aut $\underline{P}_{n}$. For any $f \in K$ there exists $u: \underline{D}(\underline{f}) \rightarrow P_{n}$ such that $f \in U=$ Fail $_{\underline{f}}(u)$. Since $f$ is a retract of $f \leqslant$, Lemma 2.4( $(\bar{b})$ implies that $f \leqslant \in U$. Thus $\bar{W}_{H} \subseteq U$ (in fact, by minimality, $\left.W_{H}=U\right)$. If $H$ were not maximal among subgroups of Aut $\underline{P}_{n}$, we could find a strictly larger such subgroup $H_{1}$. Then $W_{H_{1}} \varsubsetneqq W_{H} \subseteq U$, contradicting the minimality of $U$. Conversely, if $H$ is as stated in the theorem, $W_{H}$ is a failset of each of its members. The maximality of $H$ ensures that it must be a minimal failset of each of the automorphisms in it. Now suppose that $f \leqslant \in W \subseteq W_{H}$ and 
$W$ is a minimal failset of $f \leqslant$. If $W$ contained no automorphism then Theorem 7.7 would imply that $W$ contains every relation isomorphic to $f \leqslant$, and so the set $\left\{g \leqslant \mid g \in\right.$ Aut $\left.\underline{P}_{n}\right\}$. This is impossible since $W \subseteq W_{H}$ and $H$ is proper. Hence $W$ must contain an automorphism, and by the same argument as above, $W=W_{H}$. Hence $W_{H}$ is a minimal failset of each of its members. By Theorem 3.14, $W_{H}$ is then globally minimal.

Note that if $f$ is an endomorphism and $g$ is an automorphism, then $\left\{g, f g^{-1}\right\} \vdash f$ (since $\left.f=\left(f g^{-1}\right) g\right)$. Consequently, any failset $U$ which contains $f$ but not $g$ must also contain $f g^{-1}$. In particular, every failset containing an automorphism must contain at least half the automorphism group.

We now have a complete description of the minimal failsets in $\mathbb{S}\left(\underline{P}_{n}^{2}\right)$.

Theorem 7.12. Let $\Omega=\mathbb{S}\left(\underline{P}_{n}\right) \cup \mathbb{S}\left(\underline{P}_{n}^{2}\right)$. Then the (globally) minimal failsets are

(a) $\Phi_{\{0,1\}}$, as described in Proposition 7.9 ,

(b) the sets $W_{H}$, where $H$ is a maximal proper subgroup of Aut $\underline{P}_{n}$, as described in Proposition 7.10, and

(c) the isomorphism classes of subalgebras maximal in $\alpha^{-1}(\leqslant)$.

The failsets of types (a) and (c) are pairwise disjoint, and no failset of type (b) intersects one of type (a). Each of the relations $f \leqslant$ and $(f \leqslant)^{\cup}$, for $f \in$ Aut $\underline{P}_{n}$, lies in a unique failset of type (c) as well as in each failset $W_{H}$, where $H$ is a maximal proper subgroup of Aut $\underline{P}_{n}$ which does not contain $f$.

Proof. We first need to ensure that there are no globally minimal failsets other than those listed. We know from the Piggyback Duality Theorem that it is possible to get a duality for $\mathbf{B}_{n}$ using any one of the endomorphisms mapping into $\{0,1\}$, a generating set for the automorphism group, and one partition-induced relation of each shape. Hence there does not exist a globally minimal failset containing none of these relations.

Since the endomorphisms together with the partition-induced relations yield a duality, any globally minimal failset $U$ not containing any partition-induced relation and not containing any automorphism must contain at least one endomorphism with codomain $\{0,1\}$. Since we may obtain an optimal duality which includes any one $\{0,1\}$-valued endomorphism, and no other relations of this type, $U$ must contain all such relations. We may also assume (Theorem 7.7 ) that $U$ contains no partitioninduced relation and no automorphism (Theorem 7.11). Suppose for a contradiction that $U$ does not contain the relation $N=\{0,1\}$. We take $U=\operatorname{Fail}_{\underline{P}_{n}}(u)$ and assume that $u$ fails to preserve every $\{0,1\}$-valued endomorphism, and preserves all partition-induced relations, all automorphisms, and $N$. Since $u$ preserves $N$, it must map every $\{0,1\}$-valued endomorphism into $N$. Also $u$ fails to preserve each $\{0,1\}$-valued endomorphism $e$, so that there exists $x \in$ End $\underline{P}_{n}$ such that $u(e \circ x) \neq e(u(x))$. If $x$ is a $\{0,1\}$-valued endomorphism, then $e \circ x=x$ and $u(e \circ x)=u(x)=e(u(x))$. Therefore there exists an automorphism $f_{e}$ of $\underline{P}_{n}$ such that $u\left(e \circ f_{e}\right) \neq e\left(u\left(f_{e}\right)\right)$. Since $u$ preserves $f_{e}$, we have $u\left(e \circ f_{e}\right) \neq\left(e \circ f_{e}\right)(u(\mathrm{id}))$. The map $e \circ f_{e}$ is a $\{0,1\}$-valued endomorphism. We deduce that there is some $\{0,1\}$ valued endomorphism $e$ such that $u(e) \neq e(u(\mathrm{id}))$. Let this be $e_{1}$, mapping the atom $a_{1}$ to 1 . Consider the partition-induced relation $r=E(g)$, where the Boolean homomorphism $g:[0, d] \rightarrow[0, d]$ is such that $g$ maps $a_{1}$ to $d$. For all $a \in \underline{P}_{n}$, we have $\left(a, e_{1}(a)\right) \in r$, so that $\left(\mathrm{id}, e_{1}\right) \in r_{D\left(\underline{P}_{n}\right)}$. Suppose that $u\left(e_{1}\right)=1$. Then $e_{1}(u(\mathrm{id}))=0$, so $u(\mathrm{id}) \ngtr a_{1}$. But $(a, 1) \in r$ only if $a \geqslant a_{1}$. Hence $r \in$ Fail $_{\underline{P}_{n}}(u)$, 
contrary to hypothesis. On the other hand, $u\left(e_{1}\right)=0$ implies $e_{1}(u(\mathrm{id}))=1$, whence $u($ id $) \geqslant a_{1}$. But $(a, 0) \in r$ only if $a \ngtr a_{1}$, so again we have $r \in$ Fail $_{\underline{P}_{n}}(u)$, contrary to hypothesis. Therefore $N$ belongs to $U$. Now invoke Theorem 6.5 . We conclude that $U=\Phi_{\{0,1\}}$.

All the assertions have now been proved except the claim that $\Phi_{N} \cap W_{H}=\varnothing$, for a maximal proper subgroup $H$ of Aut $\underline{P}_{n}$. This happens because $(d, d)$ belongs to every relation in $W_{H}$ but to no relation in $\Phi_{\{0,1\}}$.

The following description of absolutely avoidable relations is easily derived from Theorem 7.12 and its proof.

Corollary 7.13. Let $\Omega=\mathbb{S}\left(\underline{P}_{n}^{2}\right)$. Then $s \in \Omega$ is absolutely avoidable if and only if $s$ satisfies one of the following:

(a) $(d, 1) \notin s,(1, d) \notin s$, and either $s=\Delta_{\underline{P}_{n}}$ or $\pi_{i}(s) \notin\left\{N, P_{n}\right\} \quad(i=1,2)$;

(b) $(d, d),(1, d) \in s,(d, 1) \notin s$, and $\pi_{2}(s) \neq P_{n}$;

(c) $(d, d) \in s,(d, 1) \in s,(1, d) \notin s$, and $\pi_{1}(s) \neq P_{n}$;

(d) $(d, 1) \in s,(1, d) \in s$.

Note that all subalgebras of $\underline{P}_{n}$ other than $\{0,1\}$ and all proper partial automorphisms of $\underline{P}_{n}$ other than $\mathrm{id}_{\{0,1\}}$ are absolutely avoidable.

If we wish to set up an optimal duality using relations of minimum arity, then we choose the unary relation $\{0,1\}$ instead of one of the other relations in $\Phi_{\{0,1\}}$. Observe that, once this is done, all the remaining relations occurring in an optimal duality, up to converses, are either maximal subalgebras of $\alpha^{-1}(\leqslant)$ or are automorphisms. Thus we see that, in a strong sense, all optimal dualities for the varieties $\mathbf{B}_{n}$ are derived by the piggyback method.

In conclusion we review the cases $n=1,2$ and 3. For $n=1$ there is an essentially unique duality using relations of minimum arity, namely that in which we take the unary relation $\{0,1\}$ and the binary relation $\preccurlyeq$ (or its converse). For $n=2$, we previously obtained an optimal duality with 4 relations: two partitioninduced relations, $\preccurlyeq$ and $\dashv$, the non-identity automorphism, $f$, of $\underline{P}_{2}$, and one of the endomorphisms of $\underline{P}_{2}$ with codomain $\{0,1\}$. We now see that the last relation may be replaced by $\{0,1\}$, and that we may reduce the number of relations to 3 , by taking $f \cdot \preccurlyeq$ and $\dashv$ in place of $f, \preccurlyeq$ and $\dashv$. For $n=3$, we have four maximal proper subgroups of $S_{3}$, namely the unique subgroup of order 3 and each subgroup of order 2. Any transversal of the complements of these subgroups contains a generating set for Aut $\underline{P}_{3}$. Using relations which are at most binary, the smallest set yielding an optimal duality has 5 members, one fewer than the set $R$ obtained when we assume that $R$ contains a generating set for End $\underline{P}_{3}$. This is not at variance with Theorem 4.1 of [9], since our reduction cannot be achieved using only the left-packed relations demanded in that theorem.

Rather than producing a duality for a quasivariety $\mathcal{A}$, we are often interested in producing full or strong dualities in the sense of Clark and Davey [1]. The NU Strong Duality Theorem of [1] tells us that any duality for $\mathbf{B}_{n}$ can be upgraded to a strong duality by adding to the structure on $\underset{\sim}{P_{n}}$ a set $G$ of endomorphisms along with a set $H$ of partial endomorphisms which together compose to yield all the non-extendable partial endomorphisms of $\underline{P}_{n}$. (See Theorem 5.3 of [1].) The results of Davey, Haviar and Priestley [5] imply that, if we wish to use unary maps, we must add such sets $G$ and $H$ of operations and partial operations in order to obtain a strong duality. Thus $\preccurlyeq$ along with $e$ yields an optimal strong duality on 
$\mathbf{B}_{1}$, while the relations $\preccurlyeq$ and $\dashv$ along with the non-identity automorphism and one $\{0,1\}$-valued endomorphism yield an optimal strong duality on $\mathbf{B}_{2}$. The duality given in [9] for $\mathbf{B}_{3}$ involves 3 binary relations, two automorphisms and one proper endomorphism. To upgrade it to an optimal strong duality for $\mathbf{B}_{3}$ it suffices to add any one of the nine non-extendable partial endomorphisms of $\underline{P}_{3}$ to the structure on $\underset{\sim}{P}$, since it along with the automorphisms generates the other eight.

\section{REFERENCES}

1. D.M. Clark and B.A. Davey, The quest for strong dualities, J. Austral. Math. Soc. (Series A) 58 (1995), 248-280. MR 96d:08010

2. D.M. Clark and B.A. Davey, Natural dualities for the working algebraist, in preparation, Cambridge University Press.

3. D.M. Clark and B.A. Davey, When is a natural duality 'good'?, Algebra Universalis (to appear).

4. B.A. Davey, Duality theory on ten dollars a day, Algebras and Orders, (I.G. Rosenberg and G. Sabidussi, eds.), NATO Advanced Study Institute Series, Series C, Vol. 389, Kluwer Academic Publishers, 1993, pp. 71-111. MR 94m:08001

5. B.A. Davey, M. Haviar and H.A. Priestley, The syntax and semantics of entailment in duality theory, J. Symbolic Logic (to appear).

6. B.A. Davey and H.A. Priestley, Generalized piggyback dualities and applications to Ockham algebras, Houston Math. J. 13 (1987), 151-197. MR 89f:06021

7. B.A. Davey and H.A. Priestley, Introduction to lattices and order, Cambridge University Press, 1990. MR 91h:06001

8. B.A. Davey and H.A. Priestley, Partition-induced natural dualities for varieties of pseudocomplemented distributive lattices, Discrete Math. 113 (1993), 41-58. MR 94i:06011

9. B.A. Davey and H.A. Priestley, Optimal natural dualities, Trans. Amer. Math. Soc. 338 (1993), 655-677. MR 93j:06011

10. B.A. Davey and H.A. Priestley, Optimal natural dualities III: a miscellany of examples, in preparation.

11. B.A. Davey and H. Werner, Dualities and equivalences for varieties of algebras, Contributions to lattice theory (Szeged, 1980), (A.P. Huhn and E.T. Schmidt, eds.), Colloq. Math. Soc. János Bolyai, Vol. 33, North-Holland, Amsterdam, 1983, pp. 101-275. MR 85c:08012

12. B.A. Davey and H. Werner, Piggyback dualities, Lectures in Universal Algebra (Szeged, 1983), (L. Szabó and A. Szendrei, eds.), Coll. Math. Soc. János Bolyai, Vol. 43, North-Holland, Amsterdam, vol. 43, 1986, pp. 61-83. MR 87i:08001

13. B.A. Davey and H. Werner, Piggyback-dualitäten, Bull. Austral. Math. Soc. 32 (1985), 1-32. MR 87d:08002

14. B. Jónsson, Algebras whose congruence lattices are distributive, Math. Scand. 21 (1967), 110-121. MR 38:5689

15. A.F. Pixley, Semi-categorical algebras II, Math. Z. 85 (1964), 169-184. MR 29:5771

16. H.A.Priestley, Natural dualities, Lattice theory and its applications - a volume in honor of Garrett Birkhoff's 80th Birthday, (K.A. Baker and R. Wille, eds.), Heldermann Verlag, 1995.

17. H.A. Priestley and M.P. Ward, A multi-purpose backtracking algorithm, J. Symbolic Computation 18 (1994), 1-40. CMP 1995:3

Department of Mathematics, La Trobe University, Bundoora, Victoria 3083, AusTRALIA

E-mail address: B.Davey@latrobe.edu.au

Mathematical Institute, 24/29 St. Giles, Oxford OX1 3LB, England

E-mail address: hap@maths.ox.ac.uk 